\title{
Similar Motor Cortical Control Mechanisms for Precise Limb Control during Reaching and Locomotion
}

\author{
Sergiy Yakovenko ${ }^{1,2}$ and Trevor Drew ${ }^{3,4}$ \\ ${ }^{1}$ Division of Exercise Physiology, Department of Human Performance, and ${ }^{2}$ Center for Neuroscience, West Virginia University, Morgantown, West Virginia \\ 26506, and ${ }^{3}$ Département de Neurosciences and ${ }^{4}$ Groupe de Recherche sur le Système Nerveux Central, Université de Montréal, Montreal, Quebec H3C 3J7, \\ Canada
}

Throughout the course of evolution there has been a parallel development of the complexity and flexibility of the nervous system and the skeletomuscular system that it controls. This development is particularly evident for the cerebral cortical areas and the transformation of the use of the upper limbs from a purely locomotor function to one including, or restricted to, reaching and grasping. This study addresses the issue of whether the control of reaching has involved the development of new cortical circuits or whether the same neurons are used to control both locomotion and reaching. We recorded the activity of pyramidal tract neurons in the motor cortex of the cat both during voluntary gait modifications and during reaching. All cells showed generally similar patterns of activity in both tasks. More specifically, we showed that, in many cases, cells maintained a constant temporal relationship to the activity of synergistic muscle groups in each task. In addition, in some cells the relationship between the intensity of the cell discharge activity and the magnitude of the EMG activity was equally constant during gait modifications and reaching. As such, the results are compatible with the hypothesis that the corticospinal circuits used to control reaching evolved from those used to precisely modify gait.

Key words: cat; locomotion; motor cortex; reaching; visually guided

\section{Significance Statement}

In an article in 1989, Georgopoulos and Grillner (1989) proposed that the corticospinal control mechanisms used for reaching movements in primates may have evolved from those used to control precise modifications of gait during quadrupedal locomotion. In this article, we provide a test of this hypothesis by recording the activity of individual motor cortical cells during both behaviors. Our results are compatible with the hypothesis in that they demonstrate that individual cortical neurons exhibit similar qualitative and quantitative patterns during each behavior. Beyond a general similarity of activity patterns, we show that some cortical cells have the same relative relationship, in both phase and magnitude, to the muscle activity observed in both reach and locomotor tasks.

\section{Introduction}

It is well established that the motor cortex is essential for the control of both voluntary limb movements and visually guided locomotion (Drew et al., 2008b; Kalaska, 2009; Rizzolatti et al., 2014; Drew and Marigold, 2015). Lesion experiments show that

Received May 17, 2015; revised Sept. 7, 2015; accepted Sept. 17, 2015.

Author contributions: T.D. designed research; S.Y. and T.D. performed research; S.Y. and T.D. analyzed data; T.D. wrote the paper.

This work was supported by an operating grant (MOP-9578) from the Canadian Institutes of Health Research and an infrastructure grant from the Fonds de recherche du Québec-Santé. S.Y. currently receives salary support from a National Institutes of Health CoBRE Grant P20-GM-109098. We thank M. Bourdeau, N. De Sylva, P. Drapeau, C. Gauthier, F. Lebel, and J. Soucy for technical assistance in the performance and analysis of these experiments. We also thank Dr. Elaine Chapman for helpful comments on this manuscript.

Correspondence should be addressed to Dr. Trevor Drew, Départment de Neurosciences and Groupe de Recherche sur le Système Nerveux Central, Université de Montréal, Montreal, QC H3C 3J7, Canada. E-mail: Trevor.Drew@umontreal.ca.

DOI:10.1523/JNEUROSCI.1908-15.2015

Copyright $\odot 2015$ the authors $\quad 0270-6474 / 15 / 3514476-15 \$ 15.00 / 0$ cats with damage or impairment of the motor cortex, pyramidal tract, or corticospinal tract show deficits in their locomotion and are unable to adapt their locomotion to challenging terrain (Liddell and Phillips, 1944; Jiang and Drew, 1996; Drew et al., 2002) or to accurately reach to a target (Martin et al., 1990; Martin and Ghez, 1991). Moreover, single-unit recording studies show increased activity in motor cortical neurons, including identified pyramidal tract neurons (PTNs), during these same, challenging tasks (Drew, 1988, 1993; Amos et al., 1990; Beloozerova and Sirota, 1993; Drew et al., 2008b). Detailed analysis of the temporal patterns of motor cortical unit activity in the cat both during locomotion (Drew, 1993) and reaching (Yakovenko et al., 2011) has led us to suggest that the motor cortex may contribute to skilled movements by modulating the phase and magnitude of sequentially activated synergistic groups of muscles (Krouchev et al., 2006; Drew et al., 2008a; Krouchev and Drew, 2013). Indeed, the idea that supraspinal pathways may act by modifying spinal 
networks that are organized to produce a limited number of synergies has been particularly well developed in work by Bizzi et al. (2000), Saltiel et al. (2001), Tresch et al. (2002), and D'Avella et al. (2003), as well as by Hart and Giszter (2004), Hart and Giszter (2010), and Giszter (2015).

The question then arises as to whether the same neurons in the motor cortex contribute to the control of muscle activity in both tasks and whether the nature of that contribution is identical in the two behaviors. In the case of locomotion, any modification of motor activity must be incorporated into the basic locomotor rhythm, and the descending control signal must be integrated with the rhythmical neural signals arising from spinal and brainstem circuits. In contrast, reaching movements are generally made from a static position, and all neural activity related to the initiation and execution of the movement is created de novo. As such, both from a behavioral viewpoint and from a consideration of neural control, they are frequently treated as two quite distinct behaviors. Nonetheless, both behaviors require a precise control over the musculature of the entire arm to ensure the appropriate intralimb coordination required to produce skilled movement, suggesting that common circuits are likely to underlie this control.

In a wider context, the question of how the neural control of reaching movements in primates may have evolved has been a question of some interest in motor control. Iwaniuk and Whishaw (2000), for example, have suggested that reaching movements in primates may be considered as behavioral homologies that have emerged from reaching movements in more primitive animals. While such evolutionary forces may well have contributed to the control of reaching in primates, particularly by brainstem pathways, the development of the corticospinal tract in mammals provided a substrate to allow a more precise and flexible control of movements. In this context, Georgopoulos and Grillner (1989) raised the possibility that the corticospinal control of reaching movements in primates may have evolved from the type of skilled locomotor movements required to modify gait to step over obstacles during quadrupedal locomotion. Although widely quoted, there is little evidence to support this hypothesis.

In the current study, we determine whether neurons in the motor cortex produce similar control signals during locomotor gait modifications and reaching movements with similar cinematic and electromyographic characteristics. The present report provides evidence that the nature of the motor cortical control of visually guided gait modifications is qualitatively and, in many cases, quantitatively, identical to that observed during reaching movements.

\section{Materials and Methods}

\section{Training and surgery}

Experiments were performed on the same two male cats, MC28 and MC29 (weight, 6.6 and $4.2 \mathrm{~kg}$, respectively) used in a previous study (Yakovenko and Drew, 2009; Yakovenko et al., 2011). The cats were trained to walk on a treadmill at a comfortable speed $(0.5 \mathrm{~m} / \mathrm{s}$ for cat MC28, $0.35 \mathrm{~m} / \mathrm{s}$ for cat MC29) and to step over obstacles attached to the moving belt (Drew, 1993). Once trained in the locomotion task, the cats were trained to stand quietly on four force platforms and, following an instruction cue, to reach forward to depress a lever with either the left or the right limb, as described previously (Schepens et al., 2008; Yakovenko et al., 2011).

Once training was completed, the cats were prepared for surgery under aseptic conditions and under general anesthesia (isoflurane $2-3 \%$ with oxygen). Pairs of braided stainless steel wires were implanted into selected forelimb and hindlimb muscles, and a recording chamber was attached to the cranium of the cat to provide access to area 4 of the pericruciate cortex. Bundles of microwire electrodes were implanted into the pyramidal tract to allow antidromic activation of PTNs. Following surgery, the cats were administered buprenorphine $(0.005 \mathrm{mg} / \mathrm{kg})$ for $3 \mathrm{~d}$ and followed a $10 \mathrm{~d}$ regimen of antibiotics. Experiments were initiated 1 week after surgery. All procedures were approved by the institutional deontology committee and followed the guidelines of the Canadian Council for the Protection of Animals.

\section{Protocol}

During experimental sessions, a glass-insulated, tungsten microelectrode was advanced into layer $\mathrm{V}$ of the motor cortex and activity was recorded from PTNs, which were identified on the basis of a constant latency to stimulation of the pyramidal tract and by collision of spontaneous spikes with the antidromically evoked spikes at the appropriate latency (Lipski, 1981). Discharge activity was initially recorded while cats walked on the treadmill and stepped over one (MC29) or two (MC28) obstacles attached to the treadmill belt. The same, cylindrical, obstacle $(10 \mathrm{~cm}$ crosssectional area) was used for both cats; in cat MC28 an additional rectangular obstacle ( $3 \mathrm{~cm}$ wide by $2 \mathrm{~cm}$ high) was used. In the latter case, the obstacles were placed $155 \mathrm{~cm}$ apart, allowing for three to four step cycles between each obstacle. After recording $\sim 20$ steps over the obstacles, the cat was transferred to the force platforms to perform 10 unimpeded reaches with each limb and then 10 reaches with each limb with an obstacle placed in different locations (Yakovenko et al., 2011). The initial recordings were always made with the obstacle located $7-8 \mathrm{~cm}$ in front of the limb and $6 \mathrm{~cm}$ above the support surface of the paw (distal location), and then subsequently with the obstacle placed at the same height but only $2 \mathrm{~cm}$ in front of the paw (proximal location). Antidromic stimulation was applied at intervals throughout the locomotion and reaching tasks to ensure that the same unit was being recorded in both tasks. Electromyographic (EMG) data were bandpass filtered between 100 and $475 \mathrm{~Hz}$ and digitized at $1 \mathrm{kHz}$. Unit activity was digitized at $100 \mathrm{kHz}$ for later off-line software discrimination using time/amplitude windows.

\section{Data analysis}

General. Data were analyzed only for reaches or steps over the obstacle with the limb contralateral to the recording site, and all muscle activity referred to in this article was recorded on the side contralateral to the recording site. Data were only included in the analysis if we recorded at least four reaches over an obstacle and four steps in which the contralateral limb was the first limb to pass over the obstacle (lead limb).

For the reach, data were aligned to the onset of the contralateral forelimb flexor, the cleidobrachialis $(\mathrm{ClB})$, which becomes active at the onset of the reach (Schepens and Drew, 2003; Yakovenko and Drew, 2009; Yakovenko et al., 2011). The phase of activity of selected muscles and of unit activity was calculated with respect to the onset of the $\mathrm{ClB}$ (see below). Data were analyzed only for reaches made over the obstacle with the limb contralateral to the recording site.

The data obtained during locomotion were also synchronized to the onset and offset of the period of activity of the $\mathrm{ClB}$, which is active throughout the period of swing (Drew, 1993). The time between two successive periods of activity in the $\mathrm{ClB}$ was defined as a step cycle. We used custom software to identify steps over the obstacle and unobstructed steps (two steps before the step over the obstacle; Drew, 1993). They were further identified according to whether the limb contralateral to the recording site was the first (lead condition) or the second (trail condition) to step over an obstacle.

Resting cell discharge frequency (used for the analysis in both tasks) was calculated as the average of the cell discharge occurring during quiet standing in the period before the instruction cue for the reach. The presence of significant peaks or troughs of activity during the transport and the lever-press phases of the movement were determined with respect to this resting rate as described in our previous publication (Yakovenko et al., 2011).

Cross-correlation. Discharge frequencies were represented as the instantaneous frequency (reciprocal of the interspike interval) and averaged to produce perievent histograms (PEHs). These histograms were synchronized to the onset of activity in the $\mathrm{ClB}$ and normalized to the average duration of the reach or the step cycle by using the method of Udo et al. (1982; see also Drew and Doucet, 1991). Cell activity during 
A
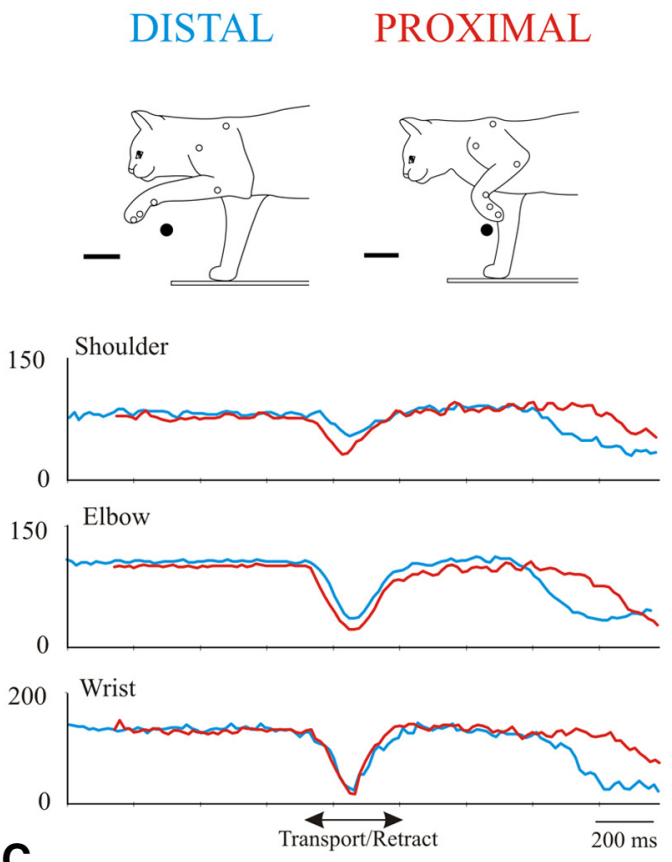

C

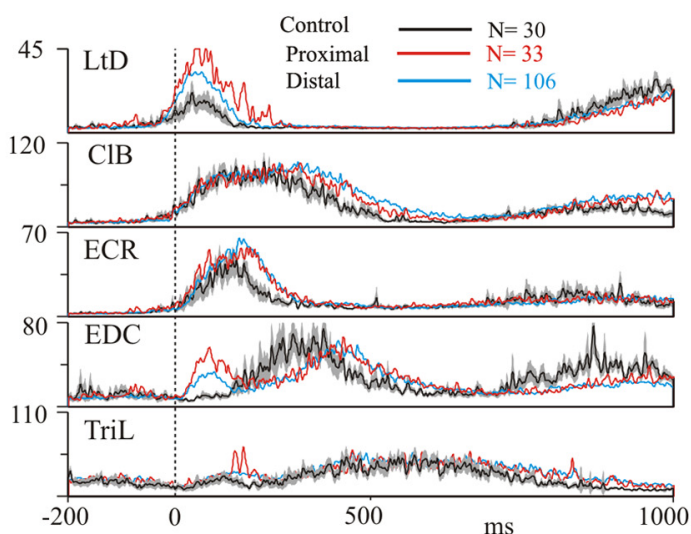

$\mathbf{E}$

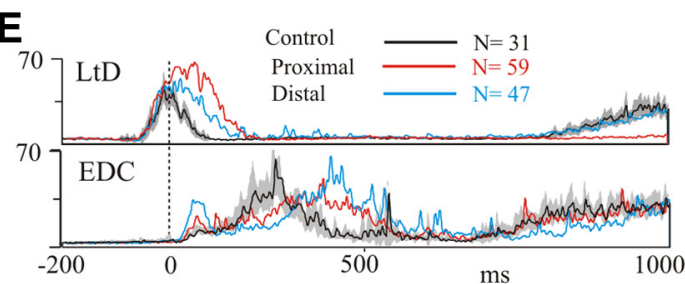

LOCOMOTION
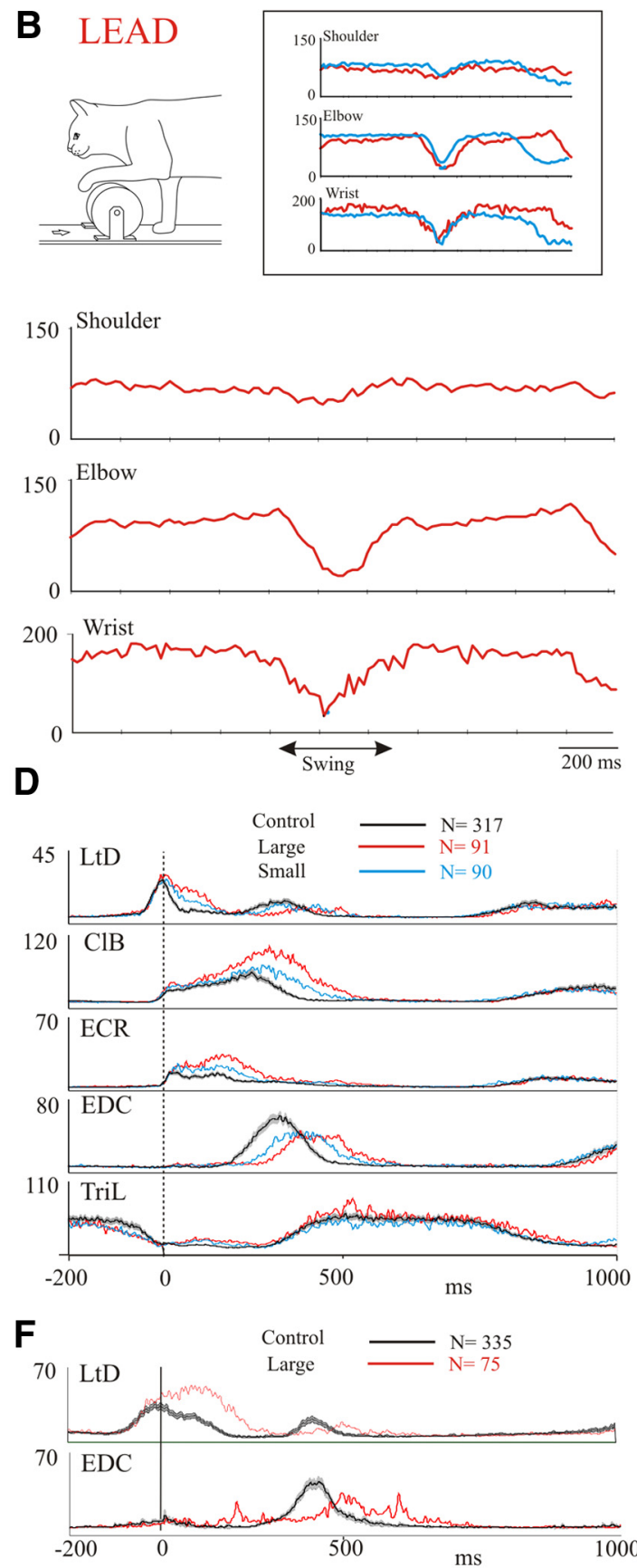

Figure 1. Comparison of the kinematics and EMG activity during reach and locomotion. $A, B$, Example joint angles for cat MC28 for the two obstacle positions (distal and proximal) used during reach $(\boldsymbol{A})$ and for a step over the large obstacle used during locomotion $(\boldsymbol{B})$. The inset directly compares the angles during locomotion over the large obstacle and during the proximal reach. $C, D$, averaged EMG activity during reach and locomotion for cat MC28. $\boldsymbol{E}, \boldsymbol{F}$, Data from two muscles from cat MC29. Data in $\boldsymbol{C}-\boldsymbol{F}$ are taken from selected days throughout the recording period of several months for each cat. In each trace, the interval of confidence $(p=0.01)$ for the unobstructed condition is shown as a gray shaded region around the average trace (black line). EMG data are expressed in arbitrary units, but are identical for reach and locomotion. Data are unfiltered and are synchronized to the onset of activity in the CIB. N indicates the number of trials included in each average for each condition.

locomotion was normalized from the onset of the $\mathrm{ClB}$ during the step over the obstacle (phase 0.0) until the onset of the next ClB burst (phase 1.0). Cell activity during reaching was normalized from the onset of activity in the $\mathrm{ClB}$ at the onset of the reach (phase 0.0) until the onset of a sustained period of activity in the shoulder retractor, the latissimus dorsi (LtD) that occurred at the end of the lever press (phase 1.0; Yakovenko et al., 2011). The traces were filtered at $50 \mathrm{~Hz}$, and the magnitude of the cell activity was normalized (value of 1.0) to the maximum peak discharge. Cross-correlations between cell discharge activity in reach and locomotion were determined using the XCORR algorithm in Matlab (MathWorks).

Phase-space analysis. Phase-space analysis was performed in a manner similar to that detailed in our previous publications (Krouchev et al., 2006; Yakovenko et al., 2011; Krouchev and Drew, 2013) to quantify the phase relationships between cell and muscle activity. As in our previous analysis (Yakovenko et al., 2011), we used five muscles to represent activity in muscle synergies active at different times during the transport phase of the reach and the swing phase of the gait modification. These 
Table 1. Comparison of the magnitude of EMG activity during reach and locomotion

\begin{tabular}{|c|c|c|c|c|}
\hline \multirow[b]{2}{*}{ Muscle } & \multicolumn{2}{|c|}{ Cat MC28 } & \multicolumn{2}{|c|}{ Cat MC29 } \\
\hline & Ratio & $p$ value & Ratio & $p$ value \\
\hline LtD & 1.11 & $<0.01$ & 0.96 & 0.26 \\
\hline CIB & 1.01 & 0.74 & 0.64 & $<0.01$ \\
\hline ECR & 1.79 & $<0.01$ & 1.57 & $<0.01$ \\
\hline EDC & 1.01 & 0.68 & 0.67 & $<0.01$ \\
\hline TriL & 0.82 & $<0.01$ & 0.69 & $<0.01$ \\
\hline
\end{tabular}

The ratio of the average EMG activity normalized to time (total integrated area/duration of the EMG burst) during reach (distal plus proximal obstacle) as a function of the activity during locomotion (large plus small obstacle in MC28, large obstacle only in MC29). The table also indicates whether the difference in magnitude was significantly different ( $p$ value) during locomotion and reach according to a two-sample $t$ test.

muscles were as follows: the shoulder retractor LtD; the shoulder protractor and elbow flexor $\mathrm{ClB}$; the wrist dorsiflexor extensor carpi radialis (ECR); the wrist and digit dorsiflexor extensor digitorum communis (EDC); and the elbow extensor, lateral head of triceps brachii (TriL).

During reach, we defined a transport phase from the onset of activity in the ClB (phase 0.0) until the end of the period of activity in the same muscle (phase 1.0). The following period, from the end of the period of activity in the $\mathrm{ClB}$ (phase 1.0) until the onset of activity in the $\mathrm{LtD}$ (phase 2.0 ) defined the lever press. Note that this is slightly different from our previous publication (Yakovenko et al., 2011) in which we used the onset of the lever press itself to subdivide the transport phase and the leverpress phase of the reach. However, the use of the $\mathrm{ClB}$ muscle as the dividing event was important to allow us to treat the locomotion and reach data in the same way. Consequently, the step cycle during locomotion was also divided into two periods based on the period of activity in the $\mathrm{ClB}$. The first period, from the onset of the activity in the $\mathrm{ClB}$ muscle to the end of the period of activity of $\mathrm{ClB}$ activity, approximated the swing period (phase $0.0-1.0$ ), and the other encompassed the remaining part of the step cycle, corresponding to stance (phase 1.0-2.0). During both reach and locomotion, the phase of the onset and offset of the periods of EMG activity in selected muscles, together with the onset and offset of bursts of cell activity was calculated with respect to the onset of the ClB. Onsets and offsets that fell between the onset and the end of the $\mathrm{ClB}$ were assigned phases between 0.0 and 1.0, while those that occurred after the offset of the $\mathrm{ClB}$ were assigned phases between 1.0 and 2.0. Outliers were identified using Tukey's method based on interquartile ranges (IQRs) and were removed from these calculations if they exceeded the upper and lower quartiles by $1.5 \times$ IQR. Overall, from the 1586 trials analyzed during locomotion and reach, this resulted in $<7 \%$ of the values being removed from any of the EMGs used in the analysis.

For the phase-space analysis, the phase of the offset of EMG or cell activity was plotted as a function of the phase of onset (Krouchev et al., 2006). Clusters were graphically illustrated with $90 \%$ confidence limits, corresponding to $2.14 \mathrm{SDs}$. In the case of the $\mathrm{ClB}$, which was used for synchronization, the onset phase value was 0.0 and the offset was 1.0 , by definition. However, in cat MC28, two recordings were made from this muscle, allowing relative phase values between the two recordings to be obtained. In MC29, in which this option was not available, we used the SDs obtained in cat MC28 for all calculations. The Euclidean distances in Figures 8 and 9 illustrate the mean distance between the center of the cell cluster and the EMG clusters. We then used the individual trials to determine whether there was a significant difference $(p<0.05, t$ test) between the distances to the two muscles with the smallest mean Euclidean distances. If there was a significant difference, the cell was classified as having a significant relationship with the closest muscle (Yakovenko et al., 2011). As an additional test, we also determined whether there was overlap between the confidence limit of the cell discharge and the confidence limit of the closest muscle in the phase space.

To compare the extent to which the phase relationship between the cell and the muscle was maintained from locomotion to reach, we calculated the distance between the vectors relating cell activity to one of the representative muscles. For this analysis, we considered that the vectors for a given relationship originated at coordinates $[0.0,0.0]$. As such, the vector provides the relative relationship between cell and muscle. The vector difference can then be calculated by simple trigonometry. For cells that had their best relationship with different muscles in each task, we applied the following two rules: (1) if both relationships (or neither) were significant, then we used the relationship between cell and muscle during the reach condition as a standard, and we then calculated the vector representing the relationship between cell and this standard muscle in the locomotion condition; and (2) if only one of the relationships was significant, we used the muscle in that task as a standard and calculated the vector for that muscle in the other condition. This analysis permitted us to determine the stability of the temporal relationship of PTNs with a given muscle. For the purposes of graphical representation only, the origins of the vectors were considered to be the centroid of the muscle with which the cell was best related, as calculated during locomotion.

To determine the probability of the distribution of vector distances occurring by chance, we used a bootstrapping protocol (Efron and Tibshirani, 1986). In brief, for each cell we shuffled the vector end point during reach with respect to that for locomotion. We passed through the entire dataset using a replacement protocol so that data for a given cell could be used more than once. We then calculated the average vector magnitude for the shuffled dataset. This bootstrap procedure was repeated 2000 times. These data were then compared with the data from the unshuffled data. In the latter case, we used the replacement procedure to also perform 2000 bootstrap operations. As a complementary analysis we performed a similar procedure using the difference between the average vector distance from the shuffled data minus the average vector distance from the unshuffled data. Again, this procedure was performed 2000 times.

Linear regressions. Linear regressions were calculated between the times of onset and offset of cell activity and the times of onset and offset of muscle activity for individual trials and step cycles. The phase of onset and offset varied between 0.0 and 2.0 as for the phase-space analysis. We also used linear regression analysis to determine the relationship between the intensity of the cell discharge and the magnitude of the EMG activity. Cell intensity was represented as the number of action potentials occurring during the period of activity of a given muscle. The magnitude of the EMG activity was represented as the integrated level of activity of the burst (sum of all activity in $1 \mathrm{~ms}$ bins). We included all trials in these analyses to maximize the range of values for the two variables. We used an ANCOVA (Systat 13, Systat Software) to determine whether the slopes of the relationships were significantly different.

Significance for all tests was found for $\alpha=0.05$.

\section{Histology}

Following all recordings, marking lesions of $35 \mu \mathrm{A}$ were made in selected locations before the cat being perfused per cardia with formaldehyde. The cortex was sectioned in the sagittal plane and the location of the recording tracks was identified as specified in Yakovenko and Drew (2009).

\section{Results}

Kinematic and EMG activity during reaching and locomotion Cats performed two similar tasks that required a precise control of limb trajectory. The first was to reach over an obstacle to press on a lever. The second was to walk on a moving treadmill belt and to step over obstacles attached to that treadmill. Stepping over obstacles was superimposed over the base walking rhythm, while in reaching the limb movement was made from a static standing position. Despite the very different nature of the two tasks, the addition of the obstacles to the reach task ensured that both the kinematics of the movements and the EMG activity were generally similar in both behaviors.

This is illustrated in Figure 1, which compares kinematic and EMG activity during reaches over obstacles placed in two different locations (distal and proximal) with the activity during steps over obstacles. Both the reach and the step over the obstacle produced similar levels and durations of flexion at each major joint of the forelimb (Fig. 1A,B), as can be seen in the inset in 
A $\quad$ MC2 8

$\mathrm{LtD}$

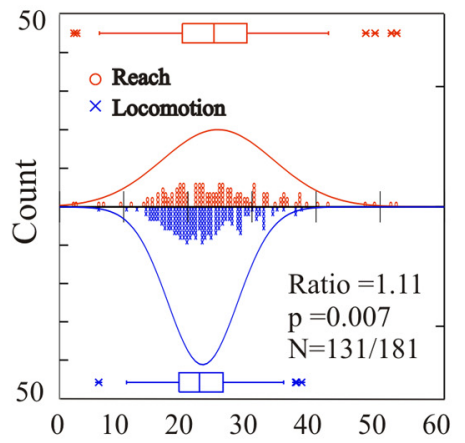

ClB

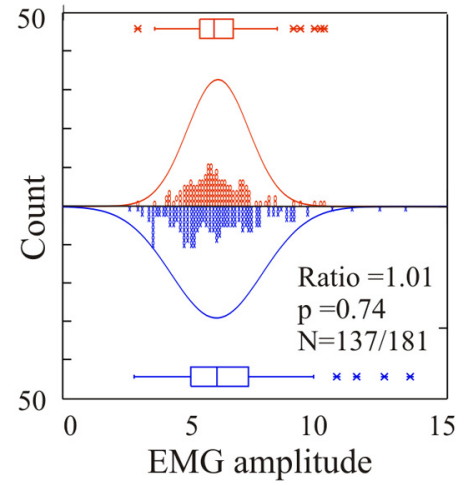

MC28

ECR

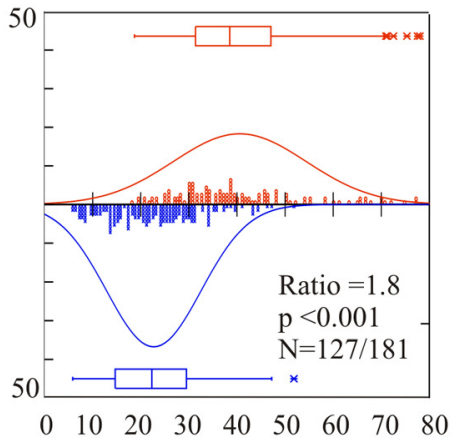

EDC

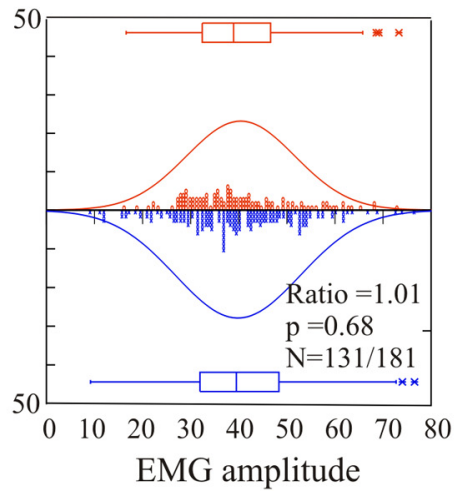

B $\quad$ MC29

LtD

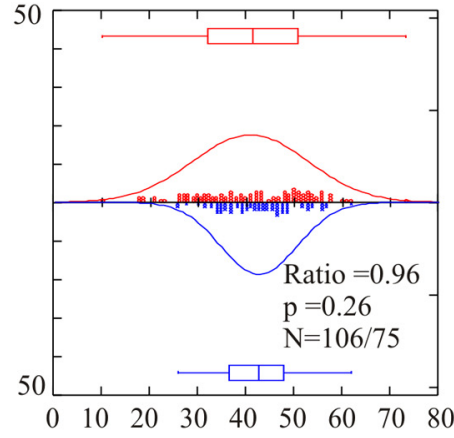

EDC

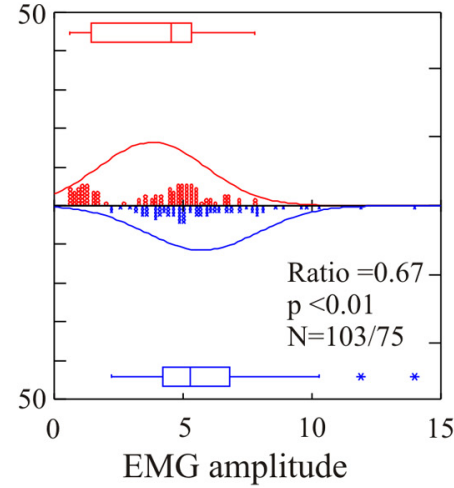

Figure 2. Comparison of EMG magnitude in the two behaviors. $\boldsymbol{A}, \boldsymbol{B}$, Comparison of the normalized magnitude of selected EMGs from cats MC28 ( $\boldsymbol{A}$ ) and MC29 ( $\boldsymbol{B})$. EMG magnitude is normalized by integrating the amplitude of the EMG in a burst of activity (1 ms bins) and then dividing by the duration of the burst. Data are illustrated in the form of histograms of EMG magnitude, density curves, and box plots in which the box indicates the mode and the first and third interquartile levels (i.e., it encompasses $50 \%$ of the values). Values for the magnitudes of the EMG are arbitrary but are comparable for the two behaviors. For each muscle, we indicate the ratio of the mean EMG magnitude during reach as a function of that during locomotion. Probability was calculated on the basis of two sample $t$ tests. Data are taken from the same trials used to create Figure 1. $N$ indicates number of trials in reach/locomotion.

Figure $1 B$. Joint angles during the proximal reach were slightly greater at the shoulder than during distal reach but were similar at the other two joints. EMG activity during the reaches over the obstacle (Fig. 1C) showed changes in the phase and the magnitude of activity when compared with the unobstructed (control) condition (Yakovenko et al., 2011). EMG activity during the steps over the obstacle showed comparable changes (Fig. 1D; Drew, 1993). Changes in EMG activity were similar in the other cat used in this study (Fig. $1 E, F$ ). Note that in this cat we had data only for the larger of the two obstacles during locomotion.

Because of the qualitative similarity of the EMG activity for the two different locations of the obstacle during reach and for the two sizes of obstacle during locomotion, these data have been combined in the analysis that follows. In addition, we restrict the analysis to the obstructed condition in each behavior because our previous studies show that neuronal activity in the motor cortex can show appreciable changes in activity between the unobstructed and obstructed conditions during locomotion (Drew, 1993; Drew et al., 1996).

The degree of similarity between the EMG activity in the two behaviors is quantified in Table 1 and Figures 2 and 3. Figure 2 displays the magnitude of the integrated EMG activity, recorded from the same trials used in Figure 1, for the four representative muscles that were active during the swing phase of locomotion and the transport phase of the reach. In most muscles, the magnitude of the activity in the two behaviors overlapped quite closely, although statistical analysis showed significant differences in the level of activity in 7 of 10 of the muscles (Table 1). Despite this, the ratio of the magnitude of the activity during locomotion and reach was close to 1.0 in many cases, with the magnitude in 6 of 10 of the muscles represented in Table 1 showing a $<30 \%$ variation in the two behaviors. The largest difference in magnitude was observed in the ECR.

The relative phase of activity in all five of our representative muscles is illustrated in Figure 3 in the form of phase-space plots. The $\mathrm{LtD}$ is the first muscle to be activated during both the transport phase of the reach and the swing phase of locomotion, and is therefore represented in the lower left of the respective plots. The EDC is activated just before the lever press ( $\sim$ phase 0.5$)$ during reach and late in swing ( phase 0.9$)$, just before foot contact in locomotion, and is represented in the middle of the plot; while the TriL is activated at approximately the same time as the EDC but discharges until end of the lever press (reach) and the end of stance (locomotion). The same sequential relationship between muscles is maintained in the two behaviors, and the only important differences reside in the relatively later onset of activity in the EDC during locomotion than during reach and in the greater variability of the muscle timing during locomotion (represented by the larger ellipses).

Together, the results from these analyses suggest that the experimental design was successful in producing both a similar pattern and magnitude of EMG activity during both the locomotion and the reaching task. As such, if individual motor cortical 
A

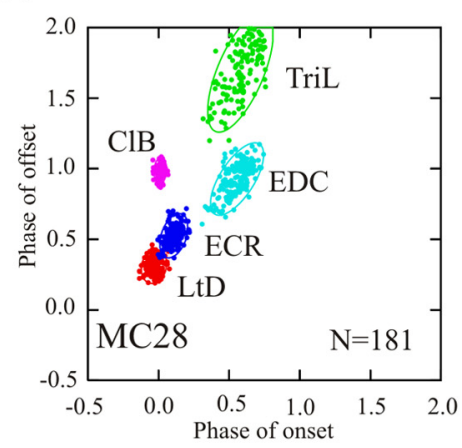

B

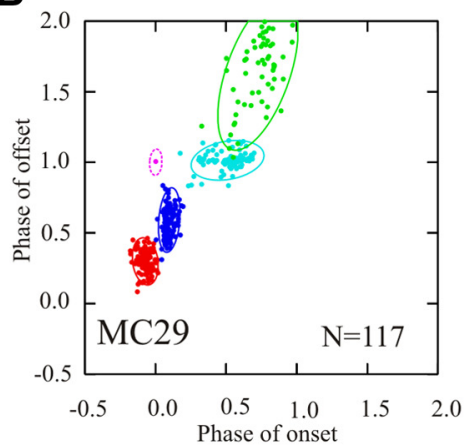

Locomotion
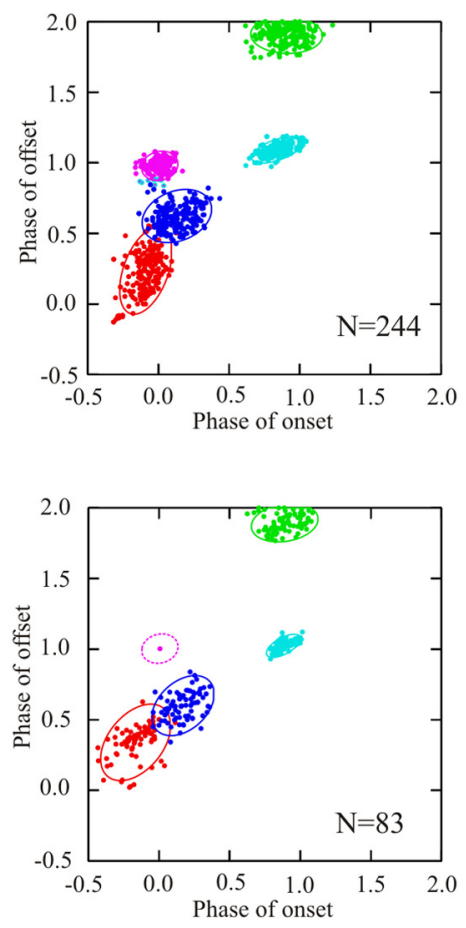

Superimposed
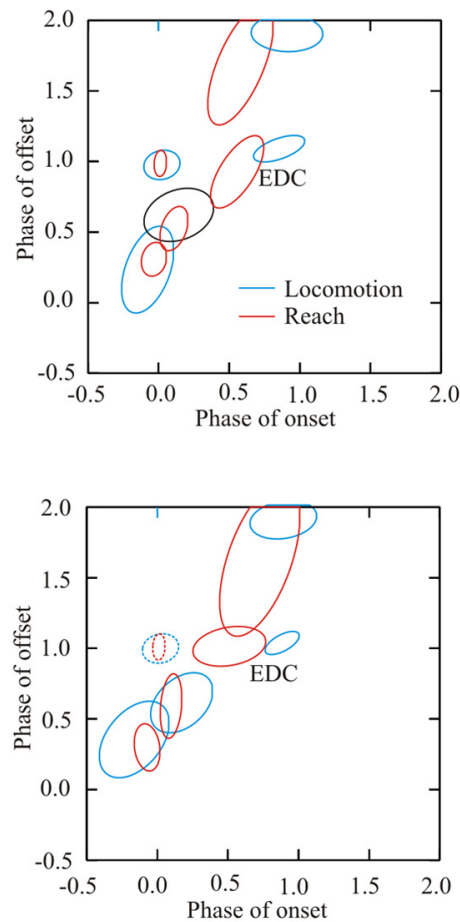

Figure 3. Phase-space analysis of EMG activity. $\boldsymbol{A}, \boldsymbol{B}$, Illustrate the period of activity of selected muscles in phase space for cats MC28 (A) and MC29 (B) by plotting the phase of offset of activity of a burst of EMG activity as a function of the phase of its onset. For each cluster of EMG bursts, we illustrate the $90 \%$ confidence limit of the period of activity. In MC29, data for the variability of the onset and offset of activity in the (IB were not available, and we therefore illustrate the variability of the data from MC28 (see Materials and Methods). Illustrations include the same trials used to create Figure 1 as well as data from two additional experiments.

cells make a similar contribution to the production of muscle activity in each task, both the phase and the magnitude of the cell activity should likewise be similar in the two tasks.

\section{Motor cortical activity during reaching and locomotion Database}

We recorded a total of 151 PTNs in the forelimb representation of the motor cortex during reaching with the contralateral forelimb (Yakovenko et al., 2011). Of these, 98 of 151 PTNs were recorded during reaches over one or both of the obstacles. We succeeded in recording neuronal activity during voluntary gait modifications from 57 of 98 of those cells that were recorded with the obstacles during reach. Our database is thus composed of those 57 cells for which we could maintain stable recordings during both the locomotion and the reaching task. Of these, 55 of 57 cells showed significant changes in activity during some phase of the reach and form the basis of the current report. Most of the cells showed a significant peak (27 of 55 cells) or trough (18 of 55 cells) of activity during the transport phase of the movement, while 15 of 55 cells (peak) and 7 of 55 cells (trough) showed a significant change of activity during the lever press. Some neurons exhibited more than one significant change.

\section{General characteristics}

Five example cells are illustrated in Figure 4. Four of the cells (Fig. $4 A-D)$ showed a significant increase in activity during the transport phase of the reach over the obstacle (Fig. $4 A-D$, left column) compared with quiet standing. These same cells showed a similar peak of activity during the swing phase of the voluntary gait modification (Fig. $4 A-D$, right column). The other illustrated cell
(Fig. 4E) showed increased activity during the lever press and during the stance phase of locomotion.

The changes in discharge frequency during movement compared with quiet standing are quantified in Figure $5 A$ for reach and in Figure $5 B$ for locomotion for the population of 55 cells. In both behaviors, the discharge frequency of the cells lies well to the left of the line of equivalence, indicating a strong increase in activity during both the reach and locomotion compared with the base level of discharge. The five cells illustrated in Figure $4 A-E$ are identified on the graph using a color code (Fig. 5A, Key). When comparing maximum discharge frequency during locomotion as a function of that during reach, Figure $5 C$ shows that only a very few cells ( 6 of 55 cells) showed a maximum discharge frequency during locomotion that was $20 \mathrm{~Hz}$ more that observed during reach. In contrast, peak discharge frequency during reach increased by $>20 \mathrm{~Hz}$ ( $\sim 10 \%$ of maximum discharge frequency) over that observed during locomotion for 24 of 55 of the cells. The other 25 of 55 cells (including those illustrated in Fig. 4A,C,D) showed a change of $<20 \mathrm{~Hz}$ between the two conditions. For a majority of cells ( 28 of 55 cells), the phase change was $<0.2$ (represented by the dotted lines), and 41 of 55 cells showed a change of phase of $<0.3$ (Fig. $5 D$ ). For comparison, the cell in Figure $4 A$ showed a phase difference for the peak activity of 0.25 , and that in Figure $4 D$ showed a difference of 0.44 (Fig. $5 D$, brown circle). In terms of time delay, the average step cycle duration in cat MC28 was $862 \pm 87 \mathrm{~ms}$, and in cat MC29 it was $1134 \pm 127 \mathrm{~ms}$, meaning that a change of phase of 0.2 


\section{Reach}

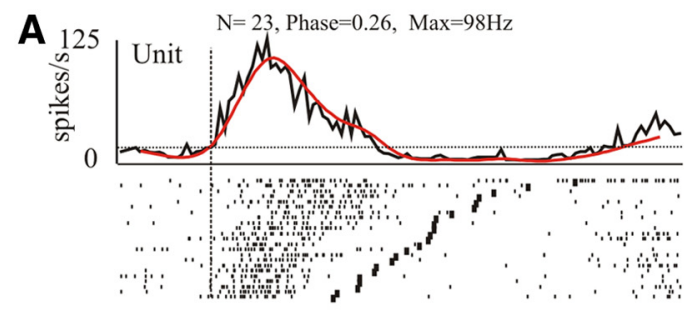

B

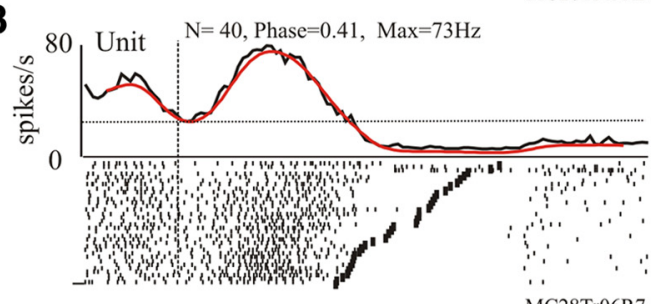

C

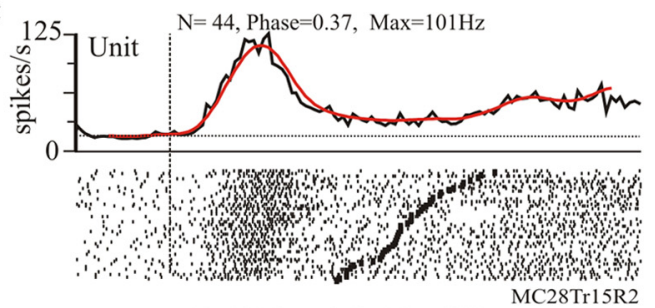

D

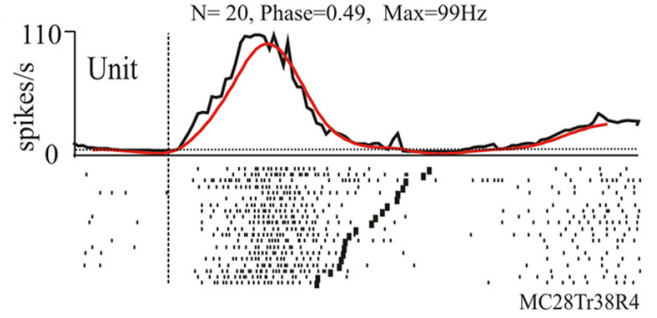

E

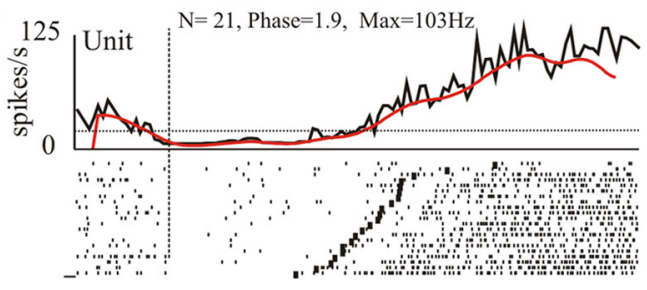

$\mathbf{F}$

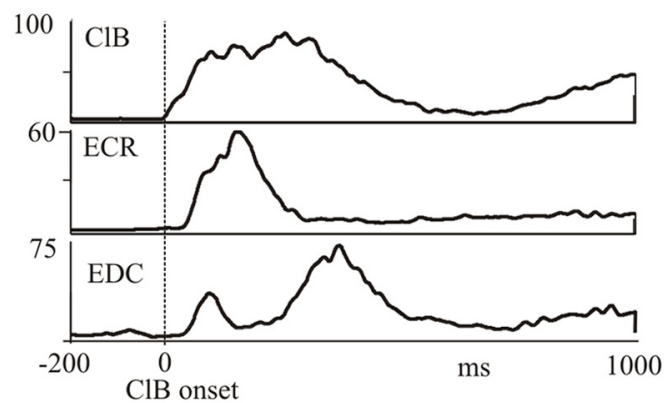

Locomotion

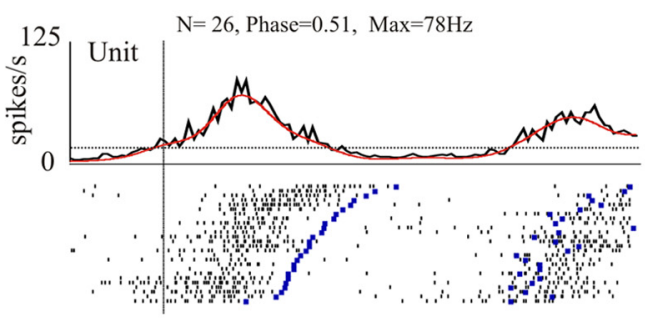

$\mathrm{MC} 28 \mathrm{Tr} 45 \mathrm{C}$
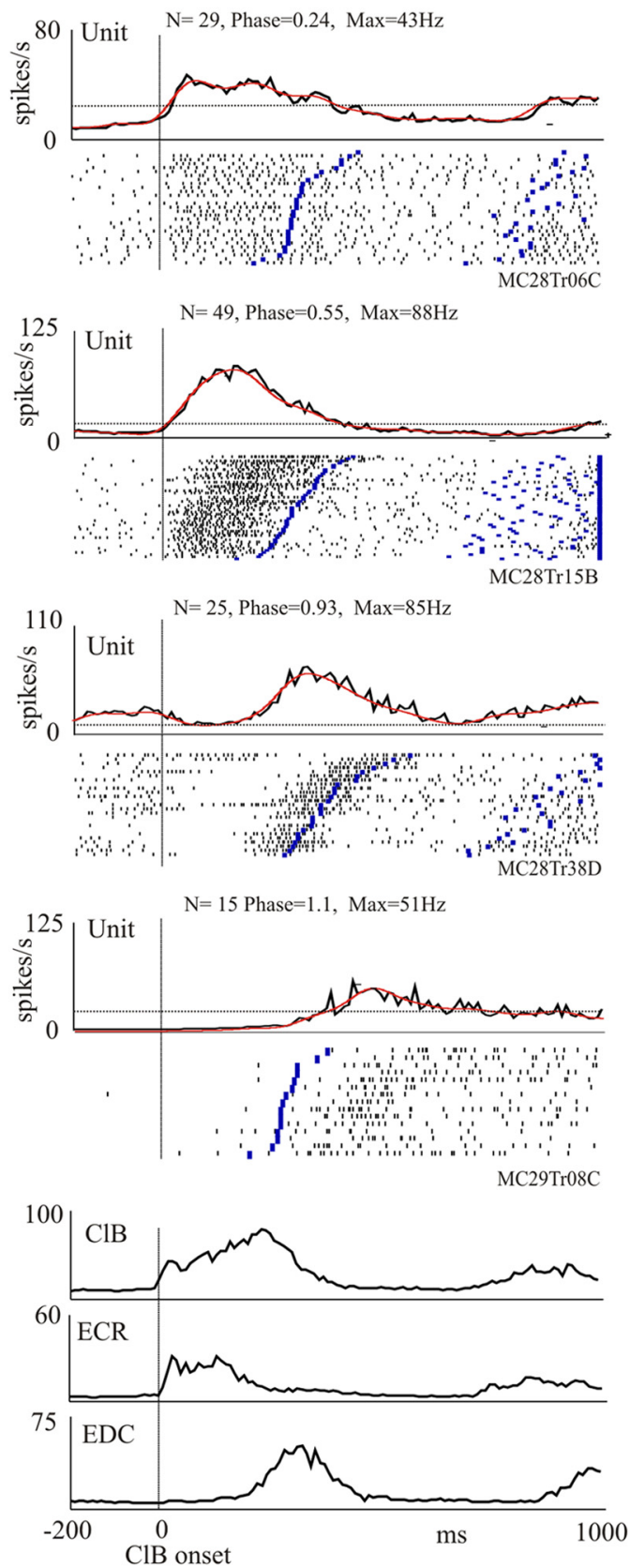

Figure 4. Activity of selected PTNs during reach and locomotion. $\boldsymbol{A}-\boldsymbol{E}$, For each of five cells, we illustrate raster displays and PEHs (black traces) of the averaged activity of the cell in the presence of an obstacle during reach (left traces) and locomotion (right traces) together with the filtered traces ( $50 \mathrm{~Hz}$, red lines). Each PEH is scaled identically for reach and locomotion. Data are synchronized to the onset of activity in the CIB (straight vertical line). The staggered lines in the rasters indicate the end of the period of activity of the CIB in each behavior. Note that the discharge activity before the (IB onset (time $=0$ ) during the reach illustrates activity during the anticipatory postural response (Yakovenko and Drew, 2009) and does not necessarily illustrate the resting (control) level of activity (see Materials and Methods), which is represented by the horizontal dotted line. Traces are not normalized, and we display activity for $200 \mathrm{~ms}$ before and $1000 \mathrm{~ms}$ after (IB onset. The bin width for the PEHs is $10 \mathrm{~ms}$. $N$, number of trials in the averages; phase and max, phase and maximum frequency of peak activity calculated from normalized and filtered traces (see Materials and Methods); F, mean EMG activity of three selected muscles recorded simultaneously with the cell illustrated in $\boldsymbol{C}$. 
A

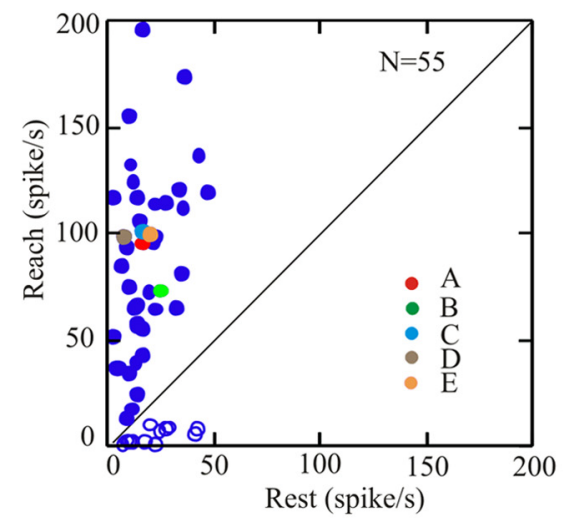

C

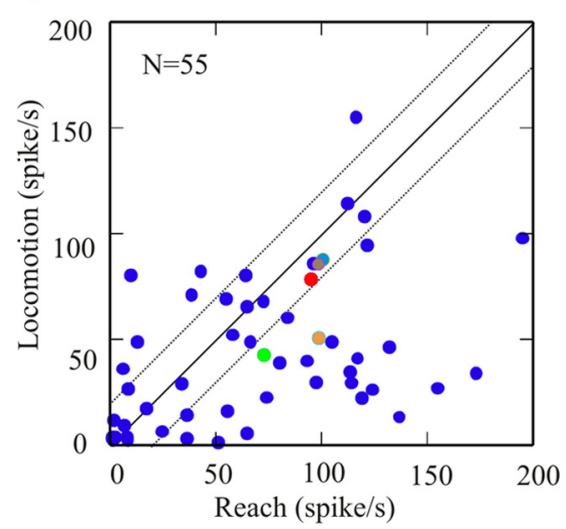

B

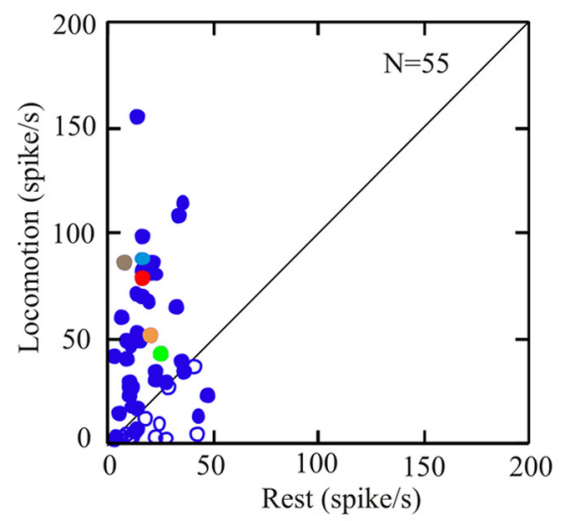

D

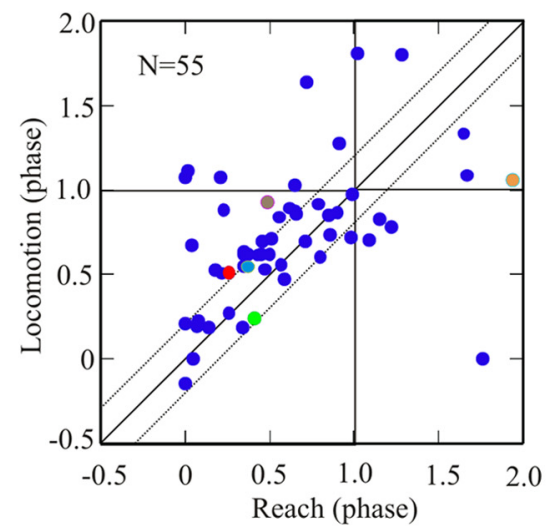

Figure 5. Comparison of maximum frequency and phase during reach and locomotion. $A, B$, Maximum frequency during reach $(\boldsymbol{A})$ and locomotion $(\boldsymbol{B})$ as a function of the resting frequency during quiet standing in 55 cells. In $\boldsymbol{A}$ and $\boldsymbol{B}$, filled circles indicate cells showing an increase in activity during reach with respect to rest (peaks); open circles indicate cells showing decreased activity (troughs). C, Maximum discharge frequency during locomotion as a function of maximum discharge frequency during reach. $\boldsymbol{D}$, Phase of maximum (or minimum for troughs) activity during locomotion as a function of the phase of activity during reach. The cells illustrated in Figure 4 are represented by colored circles, with the key provided in $\boldsymbol{A}$. $\boldsymbol{A}-\boldsymbol{D}$, Solid diagonal lines indicate the lines of equivalence. $\boldsymbol{C}, \boldsymbol{D}$, Dotted, diagonal lines show variations of $\pm 20 \mathrm{~Hz}(\boldsymbol{C})$ and \pm 0.2 phase $(\boldsymbol{D})$. All frequency and phase values are calculated from filtered traces.

corresponded to $\sim 172 \mathrm{~ms}$ in cat MC28 and $\sim 226 \mathrm{~ms}$ in cat MC29.

At a general level, these data show that individual motor cortical cells do, indeed, show similar patterns of discharge activity during the two behavioral tasks, compatible with the view that the same cells are involved in regulating muscle activity in both tasks.

\section{Quantitative analyses}

To quantitatively compare the pattern of discharge activity in the two behaviors, we used three complementary methods.

\section{Cross-correlation}

In the first place, we used cross-correlation to compare the perievent histograms of cell activity during locomotion and reach. Example results from this analysis are illustrated in Figure 6 for six cells, including, in the same order, the same 5 cells $(6 A-E)$ illustrated in Figure 4. The top three cells (Fig. 6A-C) illustrate examples in which the pattern of the discharge activity of the cell during reach and locomotion was very similar, with increased activity during swing/transport and decreased activity in stance/lever press. The peak magnitude of the crosscorrelation in these three cells occurred at, or close to, zero lag (phase $=0.0$ ), and the magnitude of the peak, at zero lag, was close to 1.0. The next example (Fig. 6D) illustrates a cell in which the general pattern of the PEHs was similar, but there were differences in the phase of activity, resulting in the phase of the peak correlation being displaced with respect to zero lag. In the last two examples illustrated (Fig. 6E,F), the cell discharge occurred at quite different phases during locomotion compared with those observed during reach, leading to a weak correlation at zero lag.

The graph and histogram of Figure 7, $A$ and $B$, respectively, summarize the results from the cross-correlation analysis for the population of 55 cells. The data in Figure $7 A$ suggest a division into two populations. In the majority of cells (39 of 55 cells), the phase of peak correlation was close to zero and the regression coefficient at zero lag exceeded 0.57 (37 of 55 cells, $>0.6$ ), suggesting that most cells showed broadly similar waveforms with at least the level of overlap illustrated by the cell in Figure $6 D$. Moreover, in 25 of 55 cells the regression coefficient at zero lag exceeded 0.7 , and in 16 of 55 cells the value exceeded 0.8 , as shown in Figure 6A-C, suggesting a very close overlap in the entire waveform of the PEH. Overall, this analysis confirms that the majority of cells showed similar activity patterns during reaching and locomotion.

The results from this analysis provide an objective confirmation of the overall similarity of the profile of the discharge patterns in the two tasks.

\section{Phase-space analysis}

The results from the cross-correlation analysis provide only a general picture of the global similarity of the patterns of discharge activity in the two behaviors. Even then, the analysis probably underestimates the similarity in the activity patterns because of the differences in the relative phase of activity of the EDC and the TriL with respect to the synchronizing muscle $(\mathrm{ClB})$ in the two behaviors (Fig. 3). For example, the phase shift in the waveforms of the cell illustrated in Figure $6 D$ is very similar to the phase difference observed in the EDC during reach and locomotion.

To obtain a more precise comparison of the phase of the discharge activity in the two conditions, we applied the same phasespace analysis as in previous publications (Krouchev et al., 2006; Yakovenko et al., 2011; Krouchev and Drew, 2013). This analysis determines whether the cell discharges in the same area of phase space as a given EMG and whether the discharge can be preferentially related to the activity of one of our representative muscles. In addition, we measured the difference between the vectors relating cell and muscle activity in the two behaviors. This latter measure provides an objective indication of the difference between the phase relations of an individual cell with an individual muscle.

The results of applying this analysis to three different cells during reach and locomotion are illustrated in Figure 8, which shows the phase relationships among three example cells and the 

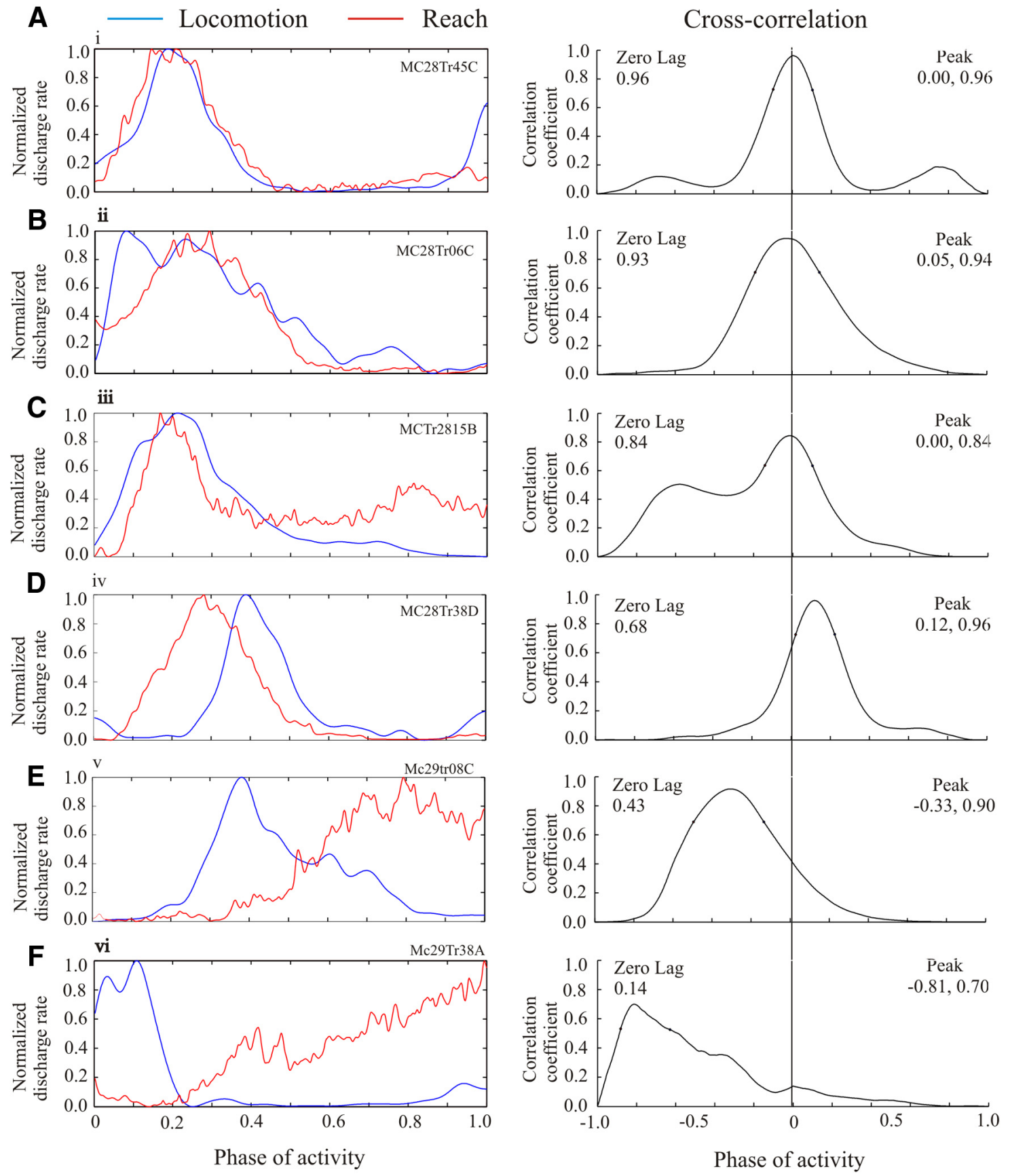

Figure 6. Cross-correlations of activity during reach and locomotion. $\boldsymbol{A}-\boldsymbol{F}$, Left column, Normalized and filtered profiles of the activity of six cells during reach (red traces) and locomotion (blue traces), including the five cells illustrated in Figure $4 A-E$. Data during locomotion are normalized to the duration of the step cycle $(0-1.0)$, while the reach data are normalized between the onset of activity in the CIB at the onset of the reach and the onset of the period of activity in the LtD as the limb was retracted (Fig. 1; Yakovenko et al., 2011). The amplitude of the traces was also normalized between 0.0 and 1.0. All traces were filtered at $50 \mathrm{~Hz}$. Right column, Cross-correlations of the activity during reach and locomotion (vertical line $=$ zero lag). $0 \mathrm{n}$ the left of the plot, we provide the magnitude of the cross-correlation at zero lag; on the right, we provide the phase (first value) and the magnitude (second value) of the peak of the cross-correlation.

five representative muscles. The first of these cells showed a preferential relationship (see Materials and Methods) with the initial period of activity of the LtD (at paw lift) both during the reach and during locomotion. The cell discharged before the activity of the LtD in both tasks was significantly better related to the LtD than to the next closest muscle, the ECR, and there was overlap of the $90 \%$ confidence limits of the cell ellipse and that of the LtD. Aligning the vectors from reach and locomotion to the centroid of the ellipse describing the mean phase-space distribution of the LtD from all experiments in cats MC28 and M29 provides a vector difference of 0.39 (phase) in the two tasks (Fig. $8 A$, right).
In some other muscles, the phase relationship was not always as stable, and cell activity was either related to different muscles in the two tasks or did not show a significant relationship with a single muscle. Figure $8 B$ (same cell as in Figs. $4 A, 6 A$ ) shows an example of this latter situation. The cell showed a significant relationship with the ECR during the reach (Fig. $8 B$, left column) but showed a nonsignificant relationship with the same muscle during locomotion (Fig. $8 B$, middle column). In the latter case, the average Euclidean distance between the centroid of the cell and the centroid of the period of ClB activity was almost the same as that between the cell and the ECR. However, aligning the vec- 
A

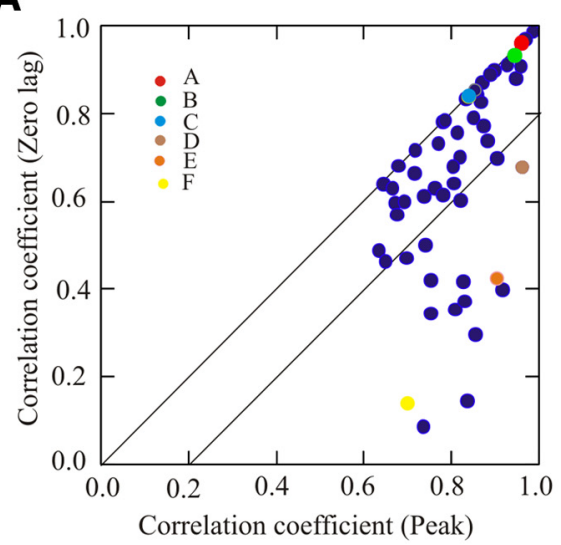

B

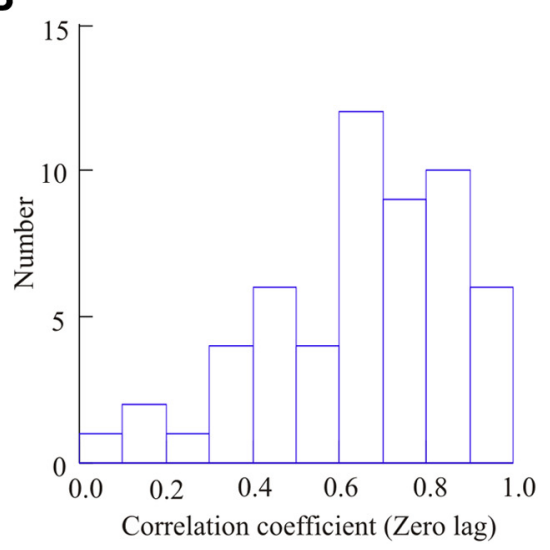

Figure 7. Quantitative analysis of the results from the cross-correlations. $\boldsymbol{A}$, Scatterplot of the magnitude of the crosscorrelation at zero-lag as a function of the maximum value of the cross-correlation. The two diagonal lines indicate the line of equivalence and $20 \%$ variation from equivalence. Colored circles indicate the cells illustrated in Figure $6 A-F$. $\boldsymbol{B}$, Histogram of the values obtained for the cross-correlation at zero-lag. $N=55$ for $\boldsymbol{A}$ and $\boldsymbol{B}$.

tors to the centroid of the average activity in the ECR (Fig. $8 B$, right) shows only a very small vector difference in the two tasks (0.07), indicating a very similar phase relationship between the cell and the ECR in both tasks, despite the lack of a preferential relationship during locomotion.

In contrast to the cell in Figure $8 B$, the cell in Figure $8 C$ (same cell as in Figs. 4D, 6D) showed its best relationship with a different muscle in reach and locomotion. During reach, the cell activity was best related to that of the $\mathrm{ClB}$, while during locomotion it was best related to the EDC. However, while the relationship with EDC was significantly different from the $\mathrm{ClB}$ during locomotion, the same was not true for reach. Moreover, aligning the cell with the centroid of the EDC produced a vector difference of only 0.13 , again indicating a very similar phase relationship to the same muscle in both tasks [for comparison, the vector difference with respect to the $\mathrm{ClB}$ for reach and locomotion was 0.47 (not illustrated)].

The result of applying the phase-space analysis to the population of cells is illustrated in Figure 9, which summarizes the phase relationships of all of those cells to which this analysis was applied during reach and locomotion. During reach (Fig. 9A), we were able to apply the analysis to 52 cells; 30 of 52 cells showed a significant (preferential) relationship with the muscle to which it was closest (smallest Euclidean distance) on the bases of the $t$ tests ( $p<0.05$; see Materials and Methods). Of these, 20 of 52 cells also showed overlap of the confidence ellipses for the cell and muscle, showing that they partially occupied the same phase space. The other 22 of 52 cells were not preferentially related to any of the muscles used in the analysis. During locomotion (Fig. $9 B$ ), we were able to apply the analysis to 51 cells; 39 of 51 cells showed a preferential relationship to one muscle, and for 23 of 51 cells there was an overlap of the confidence ellipses. A further 12 of 51 cells were not preferentially related to any of the muscles.

Altogether, 42 of 51 cells showed a preferential relationship to a given muscle during either reach or locomotion (regardless of whether the relationship was significant or not). In 20 of 42 cells, this relationship was with the same muscle for both reach and locomotion. In the other 22 of 42 cells, the relationship was between muscles that were adjacent in the phase space (Fig. $8 \mathrm{C}, \mathrm{ClB}$ and EDC). The Euclidean distances for the 20 of 42 cells with the same relationship to a given muscle are plotted in Figure $9 C$, demonstrating the similarity of the vectors for the same cells during reach (solid lines) and locomotion (dotted lines). The vector differences of these 20 cells are plotted in Figure 9D (black bars) superimposed on the values for all 42 cells. In comparison, Figure $9 E$ illustrates only those cells that showed a significant relationship in both tasks. The inset in Figure 9D illustrates all possible vector differences among the five representative muscles in each cat. Only four muscle pairs (4 of 20 pairs, 20\%) produced a vector difference of $<0.5$, while 11 of 20 pairs $(55 \%)$ of the vector differences in Figure 9D and 7 of 11 pairs (64\%) of those in Figure $9 E$ show differences of $<0.5$.

To determine a statistical measure for the probability that the vector distances are not the result of a random distribution of cell activity in phase space, we performed a bootstrapping protocol (see $\mathrm{Ma}$ terials and Methods) on the 42 cells for which data were available in both conditions. The 2000 bootstraps on the shuffled data gave a mean vector distance of 0.83 , while the corresponding value for the unshuffled data was 0.54 . The upper bound of the $95 \%$ confidence limit (two-tailed) for the unshuffled data was 0.65 , while the lower limit for the shuffled data was 0.69. There was thus complete separation between the two populations. The complementary analysis in which the bootstrap was performed on the averaged unshuffled vector distance for the 42 cells subtracted from the shuffled value gave a mean value of -0.29 . The upper $95 \%$ confidence limit was -0.15 , showing a significant difference from the null hypothesis value of 0 . The bootstrapping data therefore support the hypothesis that the phase values of the cell activity with respect to muscle activity in phase space are related.

These results extend those from the cross-correlation analysis in showing not just a similarity in general discharge activity but also that $\sim 50 \%$ of the cells showed a similar phase relationship to a given muscle.

\section{Linear regression analysis}

As a third component to our quantitative analysis, we used linear regression analyses to study the relationships between temporal and magnitude measures of cell and muscle activity. The result of applying this analysis to three representative cells is illustrated in Figure 10. Figure $10 \mathrm{~A}$ illustrates the relationship between cell discharge and muscle activity in the ECR for the cell illustrated in Figures $4 A, 6 A$, and $8 B$. There are significant relationships between cell and muscle onsets and offsets for each individual behavior and for the combined activity of the two tasks. Moreover, the slopes of each of the three relationships (the two individual tasks and the combined activity) are not significantly different from each other. There was a significant relationship between cell discharge and the integrated activity of the EMG for locomotion, but not for reach. There was also a significant relationship for the combined dataset. The ANCOVA showed that there was no significant difference among the slopes of the three linear regressions. These results indicate that the cell is contributing in a similar manner to the production of muscle activity in each task.

The cell illustrated in Figure $10 B$ (same cell as in Figs. 4D, 8C) was best related to the EDC on the basis of the phase-space analysis and shows an excellent, and almost identical, linear relationship between cell onset and offset during both locomotion and 

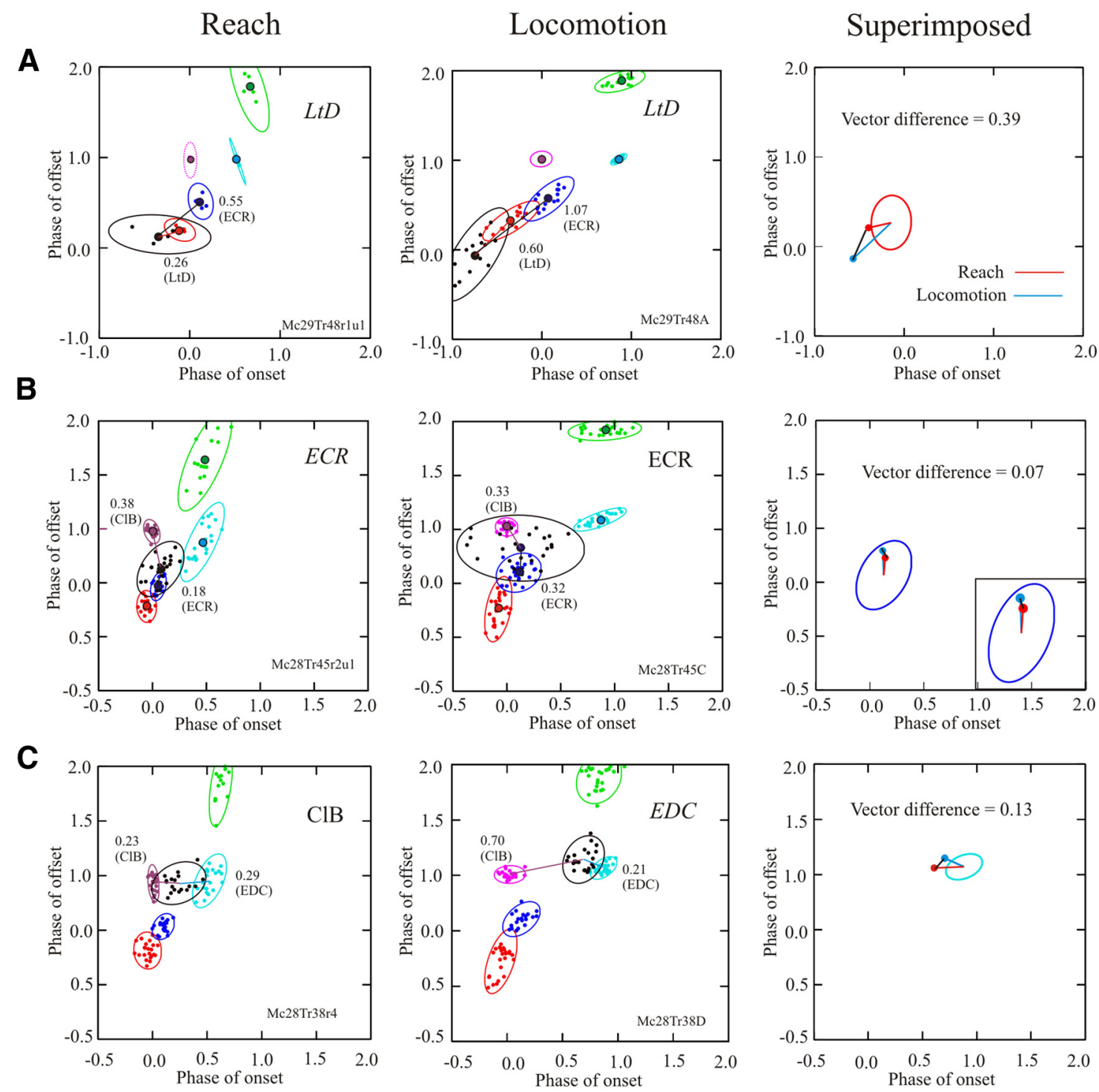

Figure 8. $\quad A-C$, Phase-space plots for three different cells during reach and locomotion. During reach (left column) and locomotion (middle column), we plot the activity of five representative muscles in the same manner as in Figure 3 . The ellipses indicate the $90 \%$ confidence limits of the distribution of the data. We also illustrate the activity of a cell (black) recorded simultaneously with the EMG activity. Colored lines and associated numerical values indicate the EMG with which the cell showed the smallest Euclidean distance, together with the EMG showing the next smallest distance. The EMG with which the cell showed the best relationship is indicated at the top right of each plot and is in italics if the relationship was significant (see Materials and Methods). In the right column, we illustrate the vectors for reach (red lines) and locomotion (blue lines) superimposed on the mean ellipses (calculated from all trials in both MC28 and MC29) of the best-related EMGs. The Euclidean distance between the end points of the two vectors (black lines) is referred to as the vector difference. Note that in $C$, the cell was best related to a different muscle for reach and locomotion. The top two cells in this figure $(\boldsymbol{A}, \boldsymbol{B})$ are also the two cells illustrated at the top of Figures 4 and 6 , and the third cell is the cell illustrated in Figures $4 D$ and $6 D$.

reach, as well as in the combined condition. The correlation between cell discharge and EMG magnitude was significant for each task as well as for the combined task, but the slope of the relationships in the two tasks was significantly different. This latter finding suggests that the cell is making a differential contribution to the level of the EMG activity in the two tasks and that it makes a proportionately greater contribution to the activity of the EDC during reach than during locomotion. In contrast, the cell in Figure $10 C$ (same as in Fig. 4C) showed a different phase-space relationship with locomotion and reach. On the basis of the phase-space analysis, the cell was best related to the ECR during reach and to the $\mathrm{ClB}$ during locomotion. The linear regression between cell activity and phase of offset was significant for both the $\mathrm{ClB}$ and the ECR in each task, but it was significant for the combined condition only for the ECR. However, the ANCOVA indicated that the slopes were significantly different for all three combinations tested and for both muscles. The relationship between the level of discharge activity and the magnitude of the EMG activity was significant only for the ECR (Fig. 10C, right), during both locomotion and reach. The ANCOVA indicated, however, that the locomotion and the reach data were from different populations, suggesting, as for the cell in Figure 10B, a differential contribution to muscle control.

Overall, we found that 29 of 55 PTNs showed a significant relationship between either the onset or offset of a burst of cell activity and the onset or offset of one of the five representative muscles during locomotion. Of these, a significant linear relationship with the muscle identified as best related on the basis of the phase analysis (Figs. 8, 9) was found for 25 of 29 cells. Similarly, during reaching 24 of 55 PTNs showed a significant, linear phase relationship with one of the five muscles, and in 
A

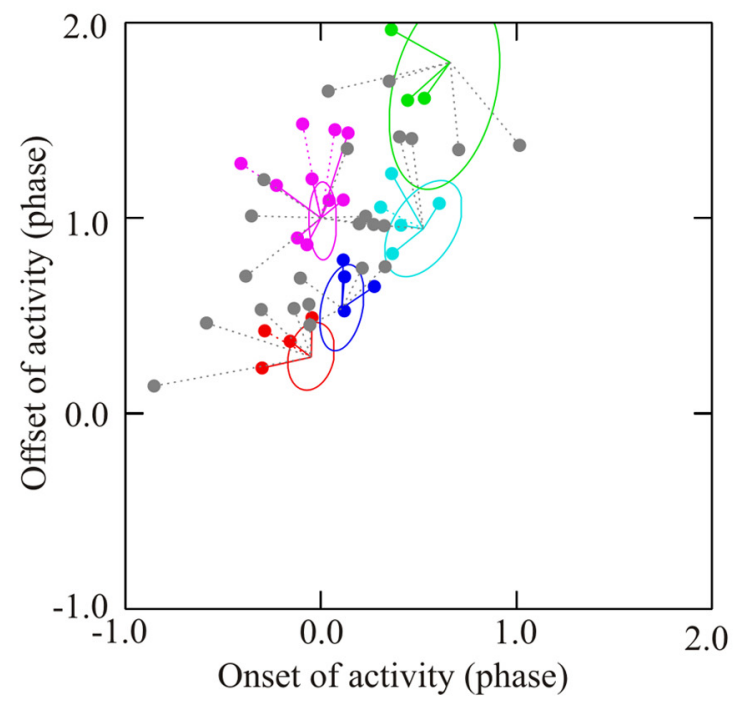

C

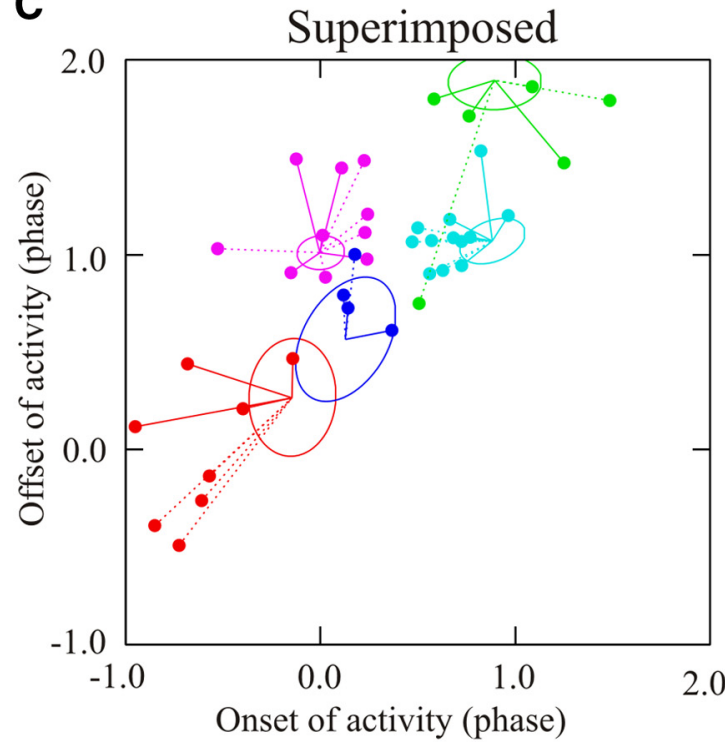

B

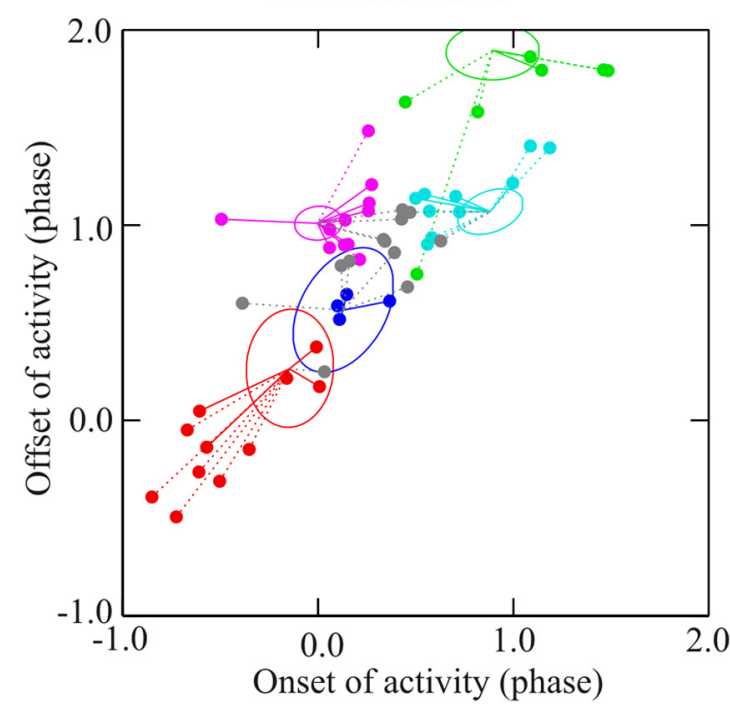

D

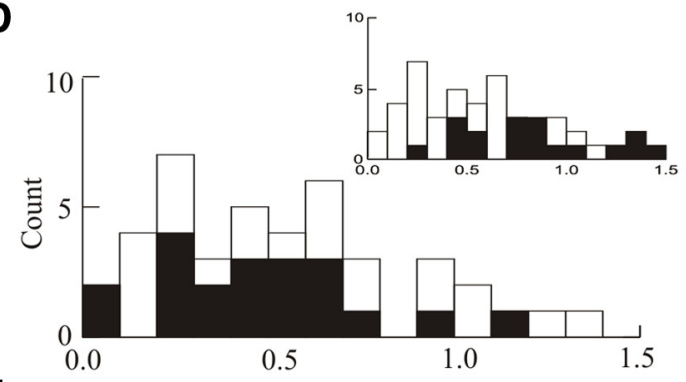

E

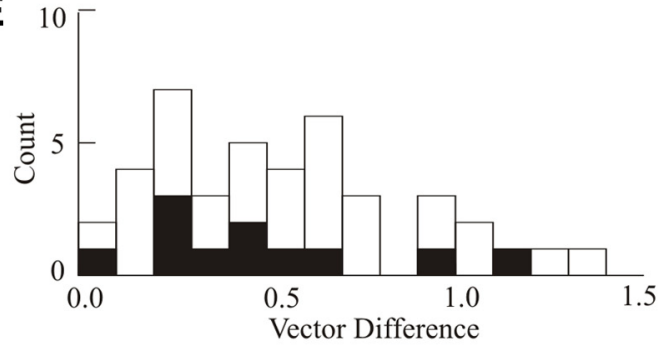

Figure 9. Phase-space analysis for all cells during reach and locomotion. $A, B$, Plots of the vectors showing the relationships between cell and muscle activity for all cells recorded during reach $(\boldsymbol{A})$ and locomotion $(\boldsymbol{B})$ for which onset and offset of cell activity could be measured on a trial-by-trial or step-by-step basis. In each plot, we indicate the following: (1) cells for which the smallest Euclidean distance was significantly different from the next smallest and for which the ellipsoid of cell activity overlapped that of the EMG with the minimum distance (colored solid lines); (2) cells for which there was a significant difference but no overlap of the ellipsoids (dotted lines); and (3) cells for which there was no significant difference between the two smallest Euclidean distances (gray circles and gray dotted lines). The latter cells are, nonetheless, linked to the muscle with which they showed the best relationship. $\boldsymbol{C}$, We superimpose the vectors for cells that showed their best relationship to the same muscle during reach (solid lines) and locomotion (dotted lines). Vectors are centered on the centroids of the EMG activity during locomotion. $D$, Vector differences for all 42 cells for which the Euclidean distance could be measured during both reach and locomotion (open bars); the filled bars illustrate the data from the 20 of 42 cells showing their best relationship to the same muscles. Inset, Vector differences for all cells, as in $\boldsymbol{D}$ (open bars), together with all combinations of the centroids of the five EMGs from each cat (filled bars). $\boldsymbol{E}$, A similar plot to that in $\boldsymbol{D}$ but with the filled bars representing vector differences for cells showing significant relationships with the same muscle in both tasks.

18 of 24 cases there was agreement with the muscle identified on the basis of the phase-space analysis. Comparing the two behaviors, 13 of 18 of the cells showing agreement with reach also maintained the same significant relationship during locomotion (Fig. 10A,B).

The results from this analysis extend those from the crosscorrelation and phase-space analyses by directly showing that some cells maintain the same phase and magnitude relationships with individual muscles in the two tasks. Moreover, this relationship is maintained even when the range of phase (Fig. 10B, left) or magnitude values (Fig. 10A, right) is different in the two tasks.

\section{Discussion}

Both locomotion and reaching movements are observed in animals without a corticospinal tract, showing that brainstem and spinal circuits have the capacity to produce complex movements (Iwaniuk and Whishaw, 2000). However, with the evolution of the motor cortex and increased flexibility in the limbs, particularly the forelimb, the corticospinal tract has become increasingly important for precision control of locomotion and reaching. The similarity of discharge patterns in the PTNs during both visually guided locomotion (Drew, 1993; Drew et al., 2008b) and reaching (Yakovenko et al., 2011) suggests that the same circuits might 
A
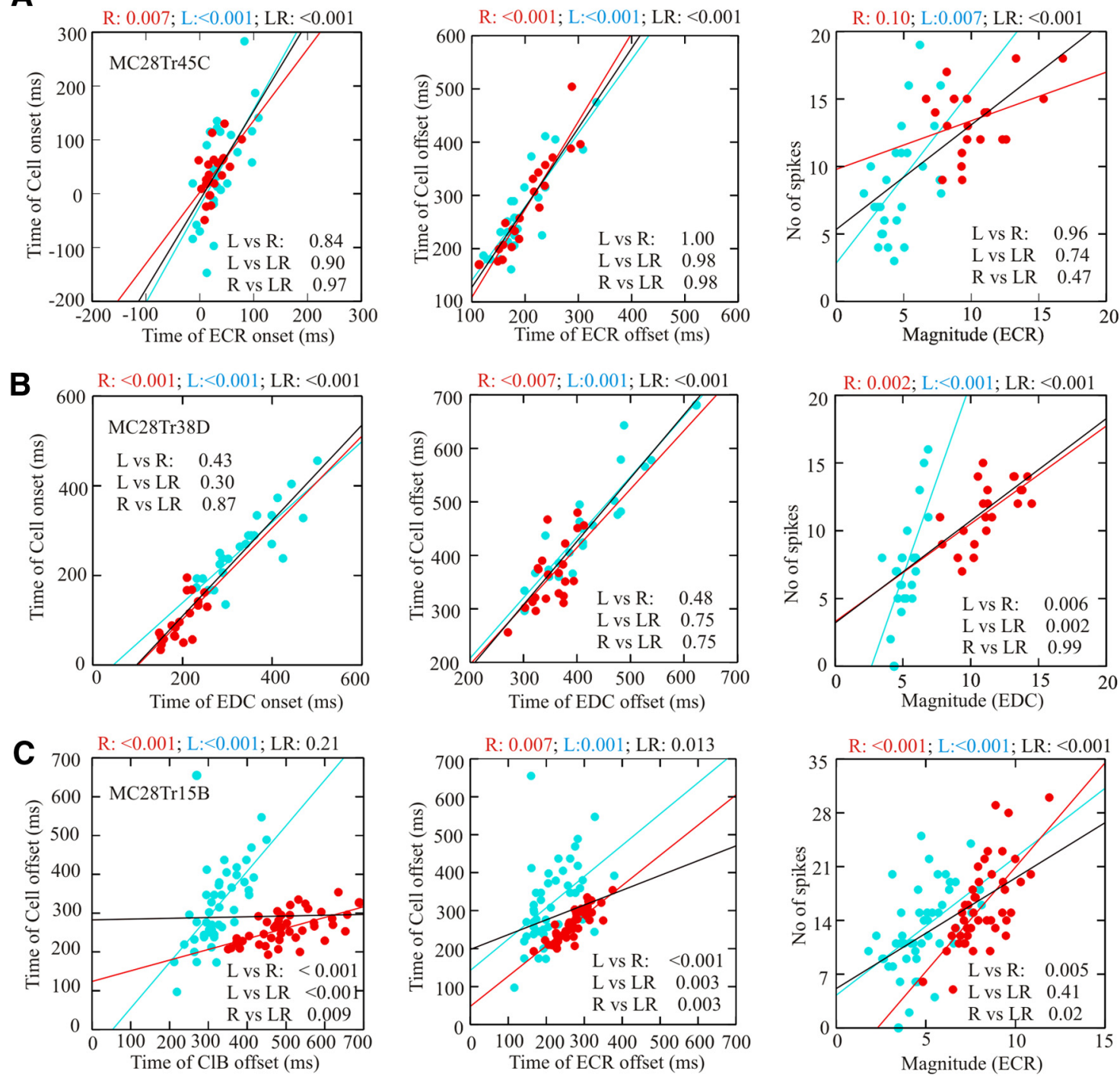

Figure 10. Linear regression analysis during reach and locomotion. $A-C$, Linear regressions for three example cells (same cells as illustrated in Figs. $4 A, D$, and $C$, respectively). Linear regressions are calculated between the time of cell onset and offset and the time of EMG onset and offset (left and middle columns) with respect to CIB onset, as well as between the number of spikes during a burst of activity and the integrated magnitude of the EMG activity (right column). The numerical values above each graph indicate the $p$ value for the reach condition alone (R, red circles and lines), the locomotion condition alone (L, cyan circles and lines), and the combined condition (LR, black lines). Numerical values within the graphs indicate the $p$ value for the ANCOVAs used to determine whether the slopes are from the same population $(p>0.05)$ or from different populations ( $L$ vs $R$, comparison of locomotion and reach; $L$ vs $L R$, comparison of locomotion and the combined data; $R$ vs $L R$, comparison of reach and the combined data).

control both behaviors. The results of this study support this view by directly showing that many PTNs in the cat motor cortex have similar task-related activity in both behaviors. This similarity extends beyond a simple propensity to discharge during both behaviors to a situation in which many cells show the same temporal and magnitude relationships to representative muscles active during both tasks. Such a close similarity in the discharge properties of the same cells in the two behaviors is fully in agreement with the hypothesis that the corticospinal control of reaching movements in primates may have evolved from precisely controlled quadrupedal locomotor movements (Georgopoulos and Grillner, 1989).

\section{Comparison of the two behaviors}

In designing the experimental paradigm to test our hypothesis, we tried to ensure that the kinematics and the level of muscle activity in the two tasks were as similar as possible by adjusting the size (and during reach, the location) of the obstacle. That we succeeded to a large extent is confirmed by the data illustrated in Figures 1, 2, and 3. In addition to similar kinematics of limb movements, we observed the same sequential, spatiotemporal progression of activity in the forelimb muscles. Moreover, the magnitude of the muscle activity was similar in both behaviors. Overall, this suggests that the pattern of EMG activity and the resultant limb trajectory required to negotiate the obstacle in the two tasks was similar, although not identical. The differences observed might reflect the differences in EMG activity required to modify an ongoing movement compared with that required to produce the movement from a stationary posture.

\section{Cell activity}

\section{General characteristics}

The general finding is that the majority of cells showed very similar discharge patterns in reach and locomotion. This observation itself is important as it demonstrates that completely separate populations of motor cortical neurons do not regulate reaching 
and locomotion. Rather, there exists a subpopulation of cells $(\sim 65 \%)$ that likely contributes in a similar manner to initiating and modifying EMG activity in each task. Although this is not totally unexpected, neither could it be predicted with complete confidence. For example, previous studies (Alstermark et al., 1981) have suggested specific control systems for reaching and locomotion at the spinal level, raising the question of whether separate populations of corticospinal neurons are also implicated. While our results do not preclude some specialization of function, they do indicate that a large proportion of the PTNs likely contributes to both behaviors. Whether the contribution of those cells with similar activity patterns to the control of behavior is mediated by segmental or propriospinal neurons cannot be determined on the basis of these experiments. However, it should be noted that the recording area in this study (histology in Yakovenko et al., 2011) overlaps the regions of the motor cortex that projects to C3-C4 propriospinal neurons (Alstermark and Ohlson, 2000a,b).

\section{Muscle-related activity}

For an important population in our database, we were able to show similar phase relationships between the cell and muscle activity, demonstrating that individual cells showed a similar relationship to the specific pattern of muscle activity required to perform the task. This is shown by the similarity in the phase space of the population (Fig. 9A,B), as well as in individual cells (Fig. 9C). In cells showing relationships to the same muscles, the vector difference between cell and muscle was relatively small and less than that predicted on the basis of a random relationship (Fig. 9D). Moreover, the bootstrap analysis showed that the vector distances between the cell representations during reach and locomotion were significantly less than would be expected by chance. Although the number of cells showing similar relationships was less than that observed with the cross-correlation analysis, this is only to be expected given the more stringent criteria used to compare the activity. Moreover, the criteria that we used to identify phase relationships probably resulted in an underestimation of the number of cells showing a constant relationship to a given muscle. This is especially true for cells showing close phase relationships to EDC as their activity frequently overlapped with that of the $\mathrm{ClB}$ during reach, but not during locomotion (Fig. $8 C$ ). In these cases, vectors differences were always smaller with respect to the EDC than to the $\mathrm{ClB}$.

The similarity of the activation patterns for some cells was even more compelling when examining the results from the linear regression analysis, which showed that some cells showed almost identical temporal relationships with the phase of onset and/or offset of a given muscle. More strikingly, several cells also showed an identical relationship between the intensity of the cell discharge and the magnitude of the muscle activity in the two tasks. This indicates that even though the discharge activity was often larger during reach than during locomotion, the cells are providing the same relative contribution to the production of the muscle activity during each task.

\section{Implications for cortical control of locomotion and reaching}

The most important finding in this study is that many motor cortical neurons make a very similar contribution to muscle activity during reaching and during stepping over an obstacle. This finding is in agreement with the proposition of Georgopoulos and Grillner (1989) that the corticospinal control circuits for independent reaching movements may have evolved from those used to make voluntary gait modifications. Although many of the cells showed greater peak discharge frequency during reaching than during locomotion (Fig. 5C), others showed similar peak activity for both tasks, and most showed similar patterns of activity and phase relationships between cell and muscle activity. Indeed, the extent of the similarity in the discharge patterns of some cells is, to some extent, surprising given that there is an underlying and fundamental difference between the two behaviors. Voluntary gait modifications are superimposed on a base locomotor rhythm that can be generated intrinsically within the spinal cord (Grillner and Zangger, 1975; Rossignol, 1996), while independent reaching movements are generated de novo from a stationary posture. As such, one might expect that the cortical contribution required to modify muscle activity that is already present and to which several structures contribute (Rossignol, 1996; Lavoie and Drew, 2002; Drew et al., 2004) would be substantially less than that required to initiate a new movement. However, while this might be true for some of the cells, others discharged as strongly during voluntary gait modifications as for reaching movements.

We believe that one reason for this is that the cortical control needed to appropriately modify the EMG patterns required to make gait modifications is not simpler than making a reach, as might be supposed, a priori, but in fact is just as challenging. As described previously (Drew, 1993; Drew et al., 2008b; Krouchev et al., 2013), the changes in EMG activity that are required to modify the trajectory of the limb are complex and precise. To produce a limb trajectory that is adjusted to the size and shape of a moving obstacle, the cat needs to modify the magnitude, duration, and temporal relationship of muscles across the forelimb. While these changes in EMG activity have to be integrated into the base locomotor pattern (Drew, 1991; Krouchev and Drew, 2013), the motor plan is, to some extent, independent of the pattern of EMG activity observed during unobstructed locomotion. In other words, the signal from the motor cortex needs to specify all the parameters of the muscle activation patterns required to step over an obstacle. As argued previously (Drew, 1993, Drew et al., 2008a,b; Lavoie et al., 2002; Krouchev and Drew, 2013), we believe that the signals required to activate small groups of synergistic muscles in a sequential manner during the swing phase are produced primarily by the motor cortex and, to a lesser extent, the red nucleus. In the same way, we have argued that the motor cortex equally plans the sequential activation of muscle activity during a reach (Yakovenko et al. 2011; see also Holdefer and Miller, 2002; Morrow et al., 2009). The challenge for the motor cortex is therefore the same in both cases, and, in retrospect, it should not be a surprise that the level of discharge activity and the contribution to the production of EMG activity is similar in the two tasks. In fact, given that the motor command during locomotion must change the base activity, one might perhaps be surprised that the activity during locomotion is not greater during this behavior than during reach.

\section{References}

Alstermark B, Ohlson S (2000a) Origin of corticospinal neurones evoking disynaptic excitation in forelimb motoneurones mediated via C3-C4 propriospinal neurones in the cat. Neurosci Res 37:91-100. CrossRef Medline

Alstermark B, Ohlson S (2000b) Origin of corticospinal neurones evoking monosynaptic excitation in C3-C4 propriospinal neurones in the cat. Neurosci Res 38:249-256. CrossRef Medline

Alstermark B, Lundberg A, Norrsell U, Sybirska E (1981) Integration in descending motor pathways controlling the forelimb in the cat. 9. Differential behavioural defects after spinal cord lesions interrupting defined pathways from higher centres to motoneurones. Exp Brain Res 42:299318. Medline 
Amos A, Armstrong DM, Marple-Horvat DE (1990) Changes in the discharge patterns of motor cortical neurones associated with volitional changes in stepping in the cat. Neurosci Lett 109:107-112. CrossRef Medline

Beloozerova IN, Sirota MG (1993) The role of the motor cortex in the control of accuracy of locomotor movements in the cat. J Physiol 461:1-25. CrossRef Medline

Bizzi E, Tresch MC, Saltiel P, d'Avella A (2000) New perspectives on spinal motor systems. Nat Rev Neurosci 1:101-108. CrossRef Medline

d'Avella A, Saltiel P, Bizzi E (2003) Combinations of muscle synergies in the construction of a natural motor behavior. Nat Neurosci 6:300-308. CrossRef Medline

Drew T (1988) Motor cortical cell discharge during voluntary gait modification. Brain Res 457:181-187. CrossRef Medline

Drew T (1991) Visuomotor coordination in locomotion. Curr Opin Neurobiol 1:652-657. CrossRef Medline

Drew T (1993) Motor cortical activity during voluntary gait modifications in the cat. I. Cells related to the forelimbs. J Neurophysiol 70:179-199. Medline

Drew T, Doucet S (1991) Application of circular statistics to the study of neuronal discharge during locomotion. J Neurosci Methods 38:171-181. CrossRef Medline

Drew T, Marigold DS (2015) Taking the next step: cortical contributions to the control of locomotion. Curr Opin Neurobiol 33:25-33. CrossRef Medline

Drew T, Jiang W, Kably B, Lavoie S (1996) Role of the motor cortex in the control of visually triggered gait modifications. Can J Physiol Pharmacol 74:426-442. CrossRef Medline

Drew T, Jiang W, Widajewicz W (2002) Contributions of the motor cortex to the control of the hindlimbs during locomotion in the cat. Brain Res Rev 40:178-191. CrossRef Medline

Drew T, Prentice S, Schepens B (2004) Cortical and brainstem control of locomotion. In: Brain mechanisms for the integration of posture and movement (Mori S, Stuart DG, Wiesendanger M, eds), pp 251-261. New York: Elsevier.

Drew T, Kalaska J, Krouchev N (2008a) Muscle synergies during locomotion in the cat: a model for motor cortex control. J Physiol 586:12391245. CrossRef Medline

Drew T, Andujar JE, Lajoie K, Yakovenko S (2008b) Cortical mechanisms involved in visuomotor coordination during precision walking. Brain Res Rev 57:199-211. CrossRef Medline

Efron B, Tibshirani R (1986) Bootstrap methods for standard errors, confidence intervals, and other measures of statistical accuracy. Stat Sci 1: 54-75. CrossRef

Georgopoulos AP, Grillner S (1989) Visuomotor coordination in reaching and locomotion. Science 245:1209-1210. CrossRef Medline

Giszter SF (2015) Motor primitives-new data and future questions. Curr Opin Neurobiol 33:156-165. CrossRef Medline

Grillner S, Zangger P (1975) How detailed is the central pattern generation for locomotion. Brain Res 88:367-371. CrossRef Medline

Hart CB, Giszter SF (2004) Modular premotor drives and unit bursts as primitives for frog motor behaviors. J Neurosci 24:5269-5282. CrossRef Medline

Hart CB, Giszter SF (2010) A neural basis for motor primitives in the spinal cord. J Neurosci 30:1322-1336. CrossRef Medline

Holdefer RN, Miller LE (2002) Primary motor cortical neurons encode functional muscle synergies. Exp Brain Res 146:233-243. CrossRef Medline

Iwaniuk AN, Whishaw IQ (2000) On the origin of skilled forelimb movements. Trends Neurosci 23:372-376. CrossRef Medline
Jiang W, Drew T (1996) Effects of bilateral lesions of the dorsolateral funiculi and dorsal columns at the level of the low thoracic spinal cord on the control of locomotion in the adult cat. 1. Treadmill walking. J Neurophysiol 76:849-866. Medline

Kalaska JF (2009) From intention to action: motor cortex and the control of reaching movements. Adv Exp Med Biol 629:139-178. CrossRef Medline

Krouchev N, Drew T (2013) Motor cortical regulation of sparse synergies provides a framework for the flexible control of precision walking. Front Comput Neurosci 7:83. CrossRef Medline

Krouchev N, Kalaska JF, Drew T (2006) Sequential activation of muscle synergies during locomotion in the intact cat as revealed by cluster analysis and direct decomposition. J Neurophysiol 96:1991-2010. CrossRef Medline

Lavoie S, Drew T (2002) Discharge characteristics of neurons in the red nucleus during voluntary gait modifications: a comparison with the motor cortex. J Neurophysiol 88:1791-1814. Medline

Liddell EGT, Phillips CG (1944) Pyramidal section in the cat. Brain 67:1-9. CrossRef

Lipski J (1981) Antidromic activation of neurones as a analytical tool in the study of the central nervous system. J Neurosci Methods 4:1-32. CrossRef Medline

Martin JH, Ghez C (1991) Impairments in reaching during reversible inactivation of the distal forelimb representation of the motor cortex in the cat. Neurosci Lett 133:61-64. CrossRef Medline

Martin JH, Priceman O, Ghez C (1990) Role of motor cortex in accuracy and coordination of prehension in the cat. Soc Neurosci Abstr 16:424.

Morrow MM, Pohlmeyer EA, Miller LE (2009) Control of muscle synergies by cortical ensembles. Adv Exp Med Biol 629:179-199. CrossRef Medline

Rizzolatti G, Cattaneo L, Fabbri-Destro M, Rozzi S (2014) Cortical mechanisms underlying the organization of goal-directed actions and mirror neuron-based action understanding. Physiol Rev 94:655-706. CrossRef Medline

Rossignol S (1996) Neural control of stereotypic limb movements. In: Handbook of physiology: Section 12. Regulation and integration of multiple systems, Ed5 (Rowell LB, Sheperd JT, eds), pp 173-216. Bethesda, MD: American Physiological Society.

Saltiel P, Wyler-Duda K, D’Avella A, Tresch MC, Bizzi E (2001) Muscle synergies encoded within the spinal cord: evidence from focal intraspinal NMDA iontophoresis in the frog. J Neurophysiol 85:605-619. Medline

Schepens B, Drew T (2003) Strategies for the integration of posture and movement during reaching in the cat. J Neurophysiol 90:3066-3086. CrossRef Medline

Schepens B, Stapley P, Drew T (2008) Neurones in the pontomedullary reticular formation signal posture and movement both as an integrated behaviour and independently. J Neurophysiol 100:2235-2253. CrossRef Medline

Tresch MC, Saltiel P, d'Avella A, Bizzi E (2002) Coordination and localization in spinal motor systems. Brain Res Rev 40:66-79. CrossRef Medline

Udo M, Kamei H, Matsukawa K, Tanaka K (1982) Interlimb coordination in cat locomotion investigated with perturbation. II. Correlates in neuronal activity of Deiter's cells of decerebrate walking cats. Exp Brain Res 46:438-447. CrossRef Medline

Yakovenko S, Drew T (2009) A Motor cortical contribution to the anticipatory postural adjustments that precede reaching in the cat. J Neurophysiol 102:853-874. CrossRef Medline

Yakovenko S, Krouchev N, Drew T (2011) Sequential activation of motor cortical neurons contributes to intralimb coordination during reaching in the cat by modulating muscle synergies. J Neurophysiol 105:388-409. CrossRef Medline 